\title{
Toeplitz $C^{*}$-Algebras on Super-Cartan Domains
}

\author{
Maribel LOAIZA and Harald UPMEIER \\ Departamento de Matemáticas \\ CINVESTAV \\ Apartado Postal 14-740 07000 \\ México D.F. - Mexico \\ mloaiza@math.cinvestav.mx \\ Fachbereich Mathematik und Informatik \\ Universität Marburg \\ 35032 Marburg - Germany \\ upmeier@mathematik. uni-marburg.de
}

Received: August 1, 2007

Accepted: December 21, 2007

\begin{abstract}
We study Hilbert spaces of super-holomorphic functions (including anti-commuting Grassmann variables) in the setting of bounded symmetric domains, more precisely for the matrix ball of arbitrary size. Our main results concern the classification of irreducible representations of the associated Toeplitz $C^{*}$ algebra and an explicit decomposition of the super-Bergman space as a direct sum of vector-valued (ordinary) Bergman spaces.
\end{abstract}

Key words: Toeplitz operator algebras, Grassmann variables, supersymmetry, Berezin quantization, Cartan domains, vector-valued Bergman spaces.

2000 Mathematics Subject Classification: 47L80, 32M15.

\section{Introduction}

Supersymmetry, involving anti-commuting Grassmann variables, plays an important role in modern mathematical physics (string theory [8], supersymmetric Standard Model, algebraic geometry (moduli spaces of super-Riemann surfaces) and analysis (fermionic Berezin integration [1], super Fock space in finite and infinite dimensions). In operator theory, besides the standard Fock space situation, the only case that has been studied in detail involves the super-symmetric versions of the so-called Cartan

Research supported by a grant from the German-Israeli Foundation (GIF), I-415-023.06/95 and by the Humboldt Foundation. 
domains, e.g. matrix domains generalizing the unit disk and the unit ball $[2,3]$. In these papers the main emphasis is on deformation quantization in the sense of Berezin, establishing the correspondence principle for Toeplitz operators on superCartan domains. The analogous result for the usual Cartan domains has been shown in [4].

On the other hand, Toeplitz operators on Cartan domains have also deep algebraic properties encoded in the $C^{*}$-algebra generated by Toeplitz operators with continuous symbols [11-13]. The present paper studies these problems, in particular the classification of all irreducible representations, for Toeplitz operators on super-Cartan domains. The main new aspect compared to the non-super case is the behavior of the fermionic (i.e., anticommuting) degrees of freedom, when passing to the representations realized on faces of the boundary. Another, somewhat surprising, result is that for domains of rank bigger than 1, the analysis of (scalar) super-Toeplitz operators involves necessarily Bergman spaces of vector-valued holomorphic functions. This gives another motivation for a deeper study of these Hilbert spaces which comprise the so-called holomorphic discrete series of the biholomorphic automorphism group.

It should be noted that the investigation of super-Cartan domains (either in the bounded realization or in the unbounded realization as generalized half-planes) is not a straightforward modification of the flat Fock space situation, since the symbol classes considered are quite different: whereas the Fock space over a super-vector space is associated with almost-periodic functions (using the underlying group structure), the Toeplitz operators on super-Cartan domains are generated by super-polynomials and their completion (super-holomorphic functions), and the crucial algebraic tool is the Jordan theoretic description of Cartan domains.

\section{Super-Bergman spaces on the unit disk}

Let $\mathcal{O}(\mathbb{B})$ denote the algebra of all holomorphic functions $\psi(z)$ on the open unit disk

$$
\mathbb{B}:=\{z \in \mathbb{C}:|z|<1\} .
$$

Let $\Lambda_{1}$ denote the complex Grassmann algebra with generator $\zeta$, satisfying the relation $\zeta^{2}=0$. Thus

$$
\Lambda_{1}=\mathbb{C}\langle 1, \zeta\rangle .
$$

The tensor product algebra

$$
\mathcal{O}\left(\mathbb{B}^{1 \mid 1}\right):=\mathcal{O}(\mathbb{B}) \otimes \Lambda_{1}=\mathcal{O}(\mathbb{B})\langle 1, \zeta\rangle
$$

consists of all "super-holomorphic" functions

$$
\Psi=\psi_{0}+\zeta \psi_{1}
$$

with $\psi_{0}, \psi_{1} \in \mathcal{O}(\mathbb{B})$. We sometimes write

$$
\Psi(z, \zeta)=\psi_{0}(z)+\zeta \psi_{1}(z)
$$


for all $z \in \mathbb{B}$.

Definition 1.1. For $\nu>1$, the weighted Bergman space

$$
H_{\nu}^{2}(\mathbb{B}):=\mathcal{O}(\mathbb{B}) \cap L^{2}\left(\mathbb{B}, d \mu_{\nu}\right)
$$

consists of all holomorphic functions on $\mathbb{B}$ which are square-integrable for the probability measure

$$
d \mu_{\nu}(z)=\frac{\nu-1}{\pi}\left(1-|z|^{2}\right)^{\nu-2} d z .
$$

Here $d z$ denotes Lebesgue measure on $\mathbb{C}$.

It is well-known $[9]$ that $H_{\nu}^{2}(\mathbb{B})$ has the reproducing kernel

$$
K_{\nu}(z, w)=(1-z \bar{w})^{-\nu}
$$

for all $z, w \in \mathbb{B}$. Let $\Lambda_{1}^{\mathbb{C}}$ denote the complex Grassmann algebra with 2 generators $\zeta$, $\bar{\zeta}$ satisfying

$$
\zeta^{2}=\bar{\zeta}^{2}=0, \quad \zeta \bar{\zeta}=-\bar{\zeta} \zeta
$$

Thus

$$
\Lambda_{1}^{\mathbb{C}}=\mathbb{C}\langle 1, \zeta, \bar{\zeta}, \bar{\zeta} \zeta\rangle=\Lambda_{1}\langle 1, \bar{\zeta}\rangle .
$$

Let $\mathcal{C}(\overline{\mathbb{B}})$ denote the algebra of continuous functions on $\overline{\mathbb{B}}$. The tensor product

$$
\mathcal{C}\left(\overline{\mathbb{B}}^{1 \mid 1}\right):=\mathcal{C}(\overline{\mathbb{B}}) \otimes \Lambda_{1}^{\mathbb{C}}=\mathcal{C}(\overline{\mathbb{B}})\langle 1, \zeta, \bar{\zeta}, \bar{\zeta} \zeta\rangle
$$

consists of all "continuous super-functions"

$$
F=f_{00}+\bar{\zeta} f_{10}+\zeta f_{01}+\bar{\zeta} \zeta f_{11},
$$

where $f_{00}, f_{10}, f_{01}, f_{11} \in \mathcal{C}(\overline{\mathbb{B}})$. The involution on $\mathcal{C}\left(\overline{\mathbb{B}}^{1 \mid 1}\right)$ is given by

$$
\bar{F}=\bar{f}_{00}+\zeta \bar{f}_{10}+\bar{\zeta} \bar{f}_{01}+\bar{\zeta} \zeta \bar{f}_{11},
$$

where $\bar{f}(z):=\overline{f(z)}$ (pointwise conjugation).

$\mathcal{C}\left(\overline{\mathbb{B}}^{1 \mid 1}\right)$ contains $\mathcal{O}\left(\mathbb{B}^{1 \mid 1}\right)$ as a subalgebra, and for $\Psi=\psi_{0}+\zeta \psi_{1} \in \mathcal{O}\left(\mathbb{B}^{1 \mid 1}\right)$ we have

$$
\bar{\Psi} \Psi=\bar{\psi}_{0} \psi_{0}+\zeta \bar{\psi}_{0} \psi_{1}+\bar{\zeta} \bar{\psi}_{1} \psi_{0}+\bar{\zeta} \zeta \bar{\psi}_{1} \psi_{1} .
$$

Given a super-function $F \in \mathcal{C}\left(\overline{\mathbb{B}}^{1 \mid 1}\right)$ of the form (2), we define its Berezin integral

$$
\int_{\mathbb{C}^{0 \mid 1}} d \zeta F:=f_{11} \in \mathcal{C}(\overline{\mathbb{B}})
$$


and

$$
\int_{\mathbb{B}^{1 \mid 1}} d z d \zeta F(z, \zeta):=\int_{\mathbb{B}} d z \int_{\mathbb{C}^{0 \mid 1}} d \zeta F(z, \zeta)=\int_{\mathbb{B}} d z f_{11}(z)
$$

Thus the "fermionic integration" is determined by the rules

$$
\int_{\mathbb{C}^{0 \mid 1}} d \zeta \cdot \zeta=\int_{\mathbb{C}^{0 \mid 1}} d \zeta \cdot \bar{\zeta}=\int_{\mathbb{C}^{0 \mid 1}} d \zeta \cdot 1=0, \quad \int_{\mathbb{C}^{0 \mid 1}} d \zeta \cdot \bar{\zeta} \zeta=1 .
$$

As an example, we have

$$
\begin{aligned}
& \int_{\mathbb{B}^{1 \mid 1}} d z d \zeta \bar{F}(z, \zeta) F(z, \zeta) \\
& \quad=\int_{\mathbb{B}} d z\left(\overline{f_{00}(z)} f_{11}(z)+\overline{f_{11}(z)} f_{00}(z)-\overline{f_{10}(z)} f_{10}(z)+\overline{f_{01}(z)} f_{01}(z)\right),
\end{aligned}
$$

which shows that the (unweighted) Berezin integral is not positive. For $\Psi=\psi_{0}+\zeta \psi_{1} \in$ $\mathcal{O}\left(\mathbb{B}^{1 \mid 1}\right)$, it follows that

$$
\int_{\mathbb{B}^{1 \mid 1}} d z d \zeta \bar{\Psi}(z, \zeta) \Psi(z, \zeta)=\int_{\mathbb{B}^{1}} d z \overline{\psi_{1}(z)} \psi_{1}(z)
$$

is positive, but not positive definite since the $\psi_{0}$ term is not present.

Definition 1.2. For any parameter $\nu>1$ the (weighted) super-Bergman space

$$
H_{\nu}^{2}\left(\mathbb{B}^{1 \mid 1}\right) \subset \mathcal{O}\left(\mathbb{B}^{1 \mid 1}\right)
$$

consists of all super-holomorphic functions $\Psi(z, \zeta)$ which satisfy the square-integrability condition

$$
(\Psi \mid \Psi)_{\nu}:=\frac{1}{\pi} \int_{\mathbb{B}^{1 \mid 1}} d z d \zeta(1-z \bar{z}-\zeta \bar{\zeta})^{\nu-1} \overline{\Psi(z, \zeta)} \Psi(z, \zeta)<+\infty .
$$

Proposition 1.3. For $\Psi=\psi_{0}+\zeta \psi_{1} \in \mathcal{O}\left(\mathbb{B}^{1 \mid 1}\right)$ we have

$$
\frac{1}{\pi} \int_{\mathbb{B}^{1 \mid 1}} d z d \zeta(1-z \bar{z}-\zeta \bar{\zeta})^{\nu-1} \bar{\Psi}(z, \zeta) \Psi(z, \zeta)=\left(\psi_{0} \mid \psi_{0}\right)_{\nu}+\frac{1}{\nu}\left(\psi_{1} \mid \psi_{1}\right)_{\nu+1},
$$

i.e., there is an orthogonal decomposition

$$
H_{\nu}^{2}\left(\mathbb{B}^{1 \mid 1}\right)=H_{\nu}^{2}(\mathbb{B}) \oplus\left[H_{\nu+1}^{2}(\mathbb{B}) \otimes \Lambda^{1}\left(\mathbb{C}^{1}\right)\right]
$$

into a sum of weighted Bergman spaces, where $\Lambda^{1}\left(\mathbb{C}^{1}\right)$ is the 1-dimensional vector space with basis vector $\zeta$. 
Proof. Consider the binomial expansion

$$
\begin{aligned}
(1-z \bar{z}-\zeta \bar{\zeta})^{\nu-1} & =(1-z \bar{z})^{\nu-1}\left(1-\frac{\zeta \bar{\zeta}}{1-z \bar{z}}\right)^{\nu-1} \\
& =(1-z \bar{z})^{\nu-1}\left(1-(\nu-1) \frac{\zeta \bar{\zeta}}{1-z \bar{z}}\right) \\
& =(1-z \bar{z})^{\nu-1}-(\nu-1)(1-z \bar{z})^{\nu-2} \zeta \bar{\zeta} \\
& =(1-z \bar{z})^{\nu-1}+(\nu-1)(1-z \bar{z})^{\nu-2} \bar{\zeta} \zeta
\end{aligned}
$$

Then we have

$$
\begin{aligned}
\frac{1}{\pi} \int_{\mathbb{B}^{1 \mid 1}} d z d \zeta(1 & -z \bar{z}-\zeta \bar{\zeta})^{\nu-1} F(z, \zeta) \\
= & \frac{1}{\pi} \int_{\mathbb{B}^{1 \mid 1}} d z d \zeta\left[(1-z \bar{z})^{\nu-1}\left(f_{00}(z)+\zeta f_{01}(z)+\bar{\zeta} f_{10}(z)+\bar{\zeta} \zeta f_{11}(z)\right)\right. \\
& \left.+(\nu-1)(1-z \bar{z})^{\nu-2} \bar{\zeta} \zeta f_{00}(z)\right] \\
= & \frac{1}{\pi} \int_{\mathbb{B}} d z\left[(1-z \bar{z})^{\nu-1} f_{11}(z)+(\nu-1)(1-z \bar{z})^{\nu-2} f_{00}(z)\right] .
\end{aligned}
$$

While this integral is still not positive on $\mathcal{C}\left(\overline{\mathbb{B}}^{1 \mid 1}\right)$, restricting to $\Psi \in \mathcal{O}\left(\mathbb{B}^{1 \mid 1}\right)$ yields a positive definite scalar product

$$
\begin{aligned}
\frac{1}{\pi} \int_{\mathbb{B}^{1 \mid 1}} d z d \zeta(1-z \bar{z}-\zeta \bar{\zeta})^{\nu-1} \bar{\Psi}(z, \zeta) \Psi(z, \zeta) \\
=\frac{1}{\pi} \int_{\mathbb{B}^{1 \mid 1}} d z d \zeta(1-z \bar{z}-\zeta \bar{\zeta})^{\nu-1}\left(\overline{\psi_{0}(z)} \psi_{0}(z)+\zeta \overline{\psi_{0}(z)} \psi_{1}(z)\right. \\
\left.+\bar{\zeta} \overline{\psi_{1}(z)} \psi_{0}(z)+\bar{\zeta} \overline{\psi_{1}(z)} \psi_{1}(z)\right) \\
=\frac{1}{\pi} \int_{\mathbb{B}} d z\left[(1-z \bar{z})^{\nu-1} \overline{\psi_{1}(z)} \psi_{1}(z)+(\nu-1)(1-z \bar{z})^{\nu-2} \overline{\psi_{0}(z)} \psi_{0}(z)\right] \\
=\frac{1}{\pi} \int_{\mathbb{B}} d z(1-z \bar{z})^{\nu-1} \overline{\psi_{1}(z)} \psi_{1}(z)+\frac{\nu-1}{\pi} \int_{\mathbb{B}} d z(1-z \bar{z})^{\nu-2} \overline{\psi_{0}(z)} \psi_{0}(z) .
\end{aligned}
$$

In view of the normalization (1), the assertion follows.

Proposition 1.4. For $\Psi=\psi_{0}+\zeta \psi_{1} \in H_{\nu}^{2}\left(\mathbb{B}^{1 \mid 1}\right)$ we have the reproducing kernel 
property

$$
\Psi(z, \zeta)=\frac{1}{\pi} \int_{\mathbb{B}^{1 \mid 1}} d w d \omega(1-w \bar{w}-\omega \bar{\omega})^{\nu-1}(1-z \bar{w}-\zeta \bar{\omega})^{-\nu} \Psi(w, \omega),
$$

i.e., $H_{\nu}^{2}\left(\mathbb{B}^{1 \mid 1}\right)$ has the reproducing kernel

$$
K_{\nu}(z, \zeta, w, \omega)=(1-z \bar{w}-\zeta \bar{\omega})^{-\nu}
$$

Proof. Analogous to (3) we have

$$
\begin{aligned}
(1-z \bar{w}-\zeta \bar{w})^{-\nu} & =(1-z \bar{w})^{-\nu}\left(1-\frac{\zeta \bar{\omega}}{1-z \bar{w}}\right)^{-\nu}=(1-z \bar{w})^{-\nu}\left(1+\nu \frac{\zeta \bar{\omega}}{1-z \bar{w}}\right) \\
& =(1-z \bar{w})^{-\nu}+\nu(1-z \bar{w})^{-\nu-1} \zeta \bar{\omega} .
\end{aligned}
$$

Together with (3) this implies

$$
\begin{aligned}
& \frac{1}{\pi} \int_{\mathbb{B}} d w \int_{\mathbb{C}^{0 \mid 1}} d \omega(1-w \bar{w}-\omega \bar{\omega})^{\nu-1}(1-z \bar{w}-\zeta \bar{\omega})^{-\nu}\left(\psi_{0}(w)+\omega \psi_{1}(w)\right) \\
&=\frac{1}{\pi} \int_{\mathbb{B}} d v \int_{\mathbb{C}^{0 \mid 1}} d \omega\left((1-w \bar{w})^{\nu-1}+(\nu-1)(1-w \bar{w})^{\nu-2} \bar{\omega} \omega\right) \\
& \quad \times\left((1-z \bar{w})^{-\nu}+\nu(1-z \bar{w})^{-\nu-1} \zeta \bar{\omega}\right)\left(\psi_{0}(w)+\omega \psi_{1}(w)\right) \\
&=\frac{1}{\pi} \int_{\mathbb{B}} d w \int_{\mathbb{C}^{0 \mid 1}} d \omega\left((1-w \bar{w})^{\nu-1} \nu(1-z \bar{w})^{-\nu-1} \zeta \bar{\omega} \omega \psi_{1}(w)\right. \\
&\left.+(\nu-1)(1-w \bar{w})^{\nu-2} \bar{\omega} \omega(1-z \bar{w})^{-\nu} \psi_{0}(w)\right) \\
&= \frac{1}{\pi} \int_{\mathbb{B}} d w\left(\nu(1-w \bar{w})^{\nu-1}(1-z \bar{w})^{-\nu-1} \psi_{1}(w) \zeta\right. \\
&\left.+(\nu-1)(1-w \bar{w})^{\nu-2}(1-z \bar{w})^{-\nu} \psi_{0}(w)\right) \\
&= \psi_{1}(z) \zeta+\psi_{0}(z)
\end{aligned}
$$

since $\psi_{1} \in H_{\nu+1}^{2}(\mathbb{B})$ and $\psi_{0} \in H_{\nu}^{2}(\mathbb{B})$.

\section{Super-Toeplitz operators on the unit disk}

For $F \in \mathcal{C}\left(\overline{\mathbb{B}}^{1 \mid 1}\right)$, the super-Toeplitz operator $T_{F}^{(\nu)}$ on $H_{\nu}^{2}\left(\mathbb{B}^{1 \mid 1}\right)$ is defined as

$$
T_{F}^{(\nu)} \Psi=P^{(\nu)}(F \Psi),
$$

where $P^{(\nu)}$ denotes the orthogonal projection onto $H_{\nu}^{2}\left(\mathbb{B}^{1 \mid 1}\right)$. 
Theorem 2.1. With respect to the decomposition $\Psi=\psi_{0}+\zeta \psi_{1}$, the super-Toeplitz operator $T_{F}^{(\nu)}$ on $H_{\nu}^{2}\left(\mathbb{B}^{1 \mid 1}\right)$ is given by the block matrix

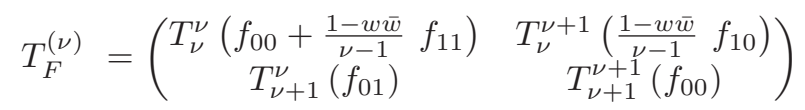

Here $T_{\nu+i}^{\nu+j}(f)$, for $0 \leq i, j \leq 1$, denotes the Toeplitz type operator from $H_{\nu+j}^{2}(\mathbb{B})$ to $H_{\nu+i}^{2}(\mathbb{B})$ defined by

$$
T_{\nu+i}^{\nu+j}(f) \psi:=P_{\nu+i}(f \psi)
$$

for $\psi \in H_{\nu+j}^{2}(\mathbb{B})$, and $P_{\nu+i}$ is the orthogonal projection from $L_{\nu+i}^{2}(\mathbb{B})$ onto $H_{\nu+i}^{2}(\mathbb{B})$.

Proof. Using (1) and (3), we obtain

$$
\begin{aligned}
& \left(T_{F}^{(\nu)} \Psi\right)(z, \zeta)=\frac{1}{\pi} \int_{\mathbb{B}} d w \int_{\mathbb{C}^{0 \mid 1}} d \omega(1-w \bar{w}-\omega \bar{\omega})^{\nu-1} \\
& \times(1-z \bar{w}-\zeta \bar{\omega})^{-\nu}\left(f_{00}(w)+\omega f_{01}(w)+\bar{\omega} f_{10}(w)+\bar{\omega} \omega f_{11}(w)\right) \\
& \times\left(\psi_{0}(w)+\omega \psi_{1}(w)\right) \\
& =\frac{1}{\pi} \int_{\mathbb{B}} d w(1-w \bar{w})^{\nu-1}(1-z \bar{w})^{-\nu} \\
& \times \int_{\mathbb{C} 0 \mid 1} d \omega\left(1+\frac{(\nu-1) \bar{\omega} \omega}{1-w \bar{w}}\right)\left(1+\frac{\nu \zeta \bar{\omega}}{1-z \bar{w}}\right) \\
& \times\left(f_{00}(w) \psi_{0}(w)+\omega\left(f_{01}(w) \psi_{0}(w)+f_{00}(w) \psi_{1}(w)\right)\right. \\
& \left.+\bar{\omega} f_{10}(w) \psi_{0}(w)+\bar{\omega} \omega\left(f_{11}(w) \psi_{0}(w)+f_{10}(w) \psi_{1}(w)\right)\right) \\
& =\frac{1}{\pi} \int_{\mathbb{B}} d w(1-w \bar{w})^{\nu-1}(1-z \bar{w})^{-\nu} \\
& \times \int_{\mathbb{C}^{0 \mid 1}} d \omega\left(\bar{\omega} \omega\left(f_{11}(w) \psi_{0}(w)+f_{10}(w) \psi_{1}(w)\right)\right. \\
& +\frac{\nu \zeta \bar{\omega}}{1-z \bar{w}} \omega\left(f_{01}(w) \psi_{0}(w)+f_{00}(w) \psi_{1}(w)\right) \\
& +\frac{(\nu-1) \bar{\omega} \omega}{1-w \bar{w}} f_{00}(w) \psi_{0}(w)=
\end{aligned}
$$




$$
\begin{aligned}
=\frac{1}{\pi} \int_{\mathbb{B}} d w(1-w \bar{w})^{\nu-1}(1-z \bar{w})^{-\nu} \\
\quad \times\left(f_{11}(w) \psi_{0}(w)+f_{10}(w) \psi_{1}(w)+\frac{\nu \zeta}{1-z \bar{w}}\left(f_{01}(w) \psi_{0}(w)\right.\right. \\
\left.\left.\quad+f_{00}(w) \psi_{1}(w)\right)+\frac{\nu-1}{1-w \bar{w}} f_{00}(w) \psi_{0}(w)\right) \\
=\frac{\nu-1}{\pi} \int_{\mathbb{B}} d w(1-w \bar{w})^{\nu-2}(1-z \bar{w})^{-\nu} f_{00}(w) \psi_{0}(w) \\
+\frac{\nu \zeta}{\pi} \int_{\mathbb{B}} d w(1-w \bar{w})^{\nu-1}(1-z \bar{w})^{-\nu-1} \\
\quad \times\left(f_{01}(w) \psi_{0}(w)+f_{00}(w) \psi_{1}(w)\right) \\
+\frac{1}{\pi} \int_{\mathbb{B}} d w(1-w \bar{w})^{\nu-2}(1-z \bar{w})^{-\nu}(1-w \bar{w}) \\
\quad \times\left(f_{11}(w) \psi_{0}(w)+f_{10}(w) \psi_{1}(w)\right) \\
=P_{\nu}\left(f_{00} \psi_{0}\right)(z)+\zeta P_{\nu+1}\left(f_{01} \psi_{0}+f_{00} \psi_{1}\right)(z) \\
+\frac{1}{\nu-1} P_{\nu}\left((1-w \bar{w})\left(f_{11} \psi_{0}+f_{10} \psi_{1}\right)\right)(z) .
\end{aligned}
$$

Theorem 2.2. The $C^{*}$-algebra $\mathcal{T}_{\nu}\left(\mathbb{B}^{1 \mid 1}\right)$, generated by all Toeplitz operators with symbols $F \in \mathcal{C}\left(\overline{\mathbb{B}}^{1 \mid 1}\right)$ which are continuous up to the boundary, can be embedded in the $2 \times 2$-matrix operator algebra

$$
\mathcal{T}_{\nu}\left(\mathbb{B}^{1 \mid 1}\right) \subset\left(\begin{array}{cc}
\mathcal{T}_{\nu}(\mathbb{B}) & \mathcal{K}_{0}^{1} \\
\mathcal{K}_{1}^{0} & \mathcal{T}_{\nu+1}(\mathbb{B})
\end{array}\right)=: \mathcal{A}
$$

where $\mathcal{T}_{\nu+i}(\mathbb{B})$ is the Toeplitz $C^{*}$-algebra on the Bergman space $H_{\nu+i}^{2}(\mathbb{B})(i=0,1)$ and $\mathcal{K}_{i}^{j}$ denotes the space of all compact operators from $H_{\nu+j}^{2}(\mathbb{B})$ to $H_{\nu+i}^{2}(\mathbb{B})$ $(i, j=0,1)$.

Proof. Realizing a super-Toeplitz operator $T_{F}^{(\nu)}$ as a $2 \times 2$-operator matrix (4), it is clear that the diagonal entries belong to the Bergman-Toeplitz $C^{*}$-algebras for $\nu$ and $\nu+1$, resp. Moreover, the off-diagonal entries are compact operators as will be shown in more detail for the more general case of the unit ball (proof of Theorem 4.2 below). Thus the embedding (5) holds on the level of generators. Since the right-hand side of $(5)$ is a $C^{*}$-algebra, the assertion follows.

The following Theorem is our main result for the case of the unit disk. 
Theorem 2.3. For every boundary point $s \in \partial \mathbb{B}$ there exists a $C^{*}$-algebra homomorphism (character)

$$
\sigma_{s}: \mathcal{T}_{\nu}\left(\mathbb{B}^{1 \mid 1}\right) \longrightarrow \mathbb{C}
$$

which is uniquely determined by the property

$$
\sigma_{s}\left(T_{f_{00}+\zeta f_{01}+\bar{\zeta} f_{10}+\bar{\zeta} \zeta f_{11}}^{(\nu)}\right)=f_{00}(s)
$$

for any continuous functions $f_{00}, f_{10}, f_{01}, f_{11} \in \mathcal{C}(\overline{\mathbb{B}})$. Moreover, the joint null-space

$$
\bigcap_{s \in \partial \mathbb{B}} \operatorname{Ker} \sigma_{s}=\mathcal{K}\left(H_{\nu}^{2}\left(\mathbb{B}^{1 \mid 1}\right)\right)
$$

of all these characters coincides with the ideal of all compact operators on the superBergman space $H_{\nu}^{2}\left(\mathbb{B}^{1 \mid 1}\right)$.

Proof. Since the Toeplitz $C^{*}$-algebra $\mathcal{T}_{\nu}(\mathbb{B})$ acts irreducibly on $H_{\nu}^{2}(\mathbb{B})$ and has a nontrivial intersection with the compact operators $\mathcal{K}\left(H_{\nu}^{2}(\mathbb{B})\right)$, it follows that $\mathcal{K}\left(H_{\nu}^{2}(\mathbb{B})\right)$ is contained in $\mathcal{T}_{\nu}(\mathbb{B})$. Applying [10, Theorem 4.12.32] to the special case of the unit disk and the Bergman-Toeplitz $C^{*}$-algebras for parameter $\nu>1$ and $\nu+1$, resp., we obtain $C^{*}$-algebra homomorphisms

$$
\begin{aligned}
\sigma_{s}^{\nu} & : \mathcal{T}_{\nu}(\mathbb{B}) \longrightarrow \mathbb{C}, \\
\sigma_{s}^{\nu+1} & : \mathcal{T}_{\nu+1}(\mathbb{B}) \longrightarrow \mathbb{C},
\end{aligned}
$$

whose kernel is the ideal of compact operators and satisfy

$$
\sigma_{s}^{\nu}\left(T_{f}^{\nu}\right)=\sigma_{s}^{\nu+1}\left(T_{f}^{\nu+1}\right)=f(s)
$$

whenever $f \in \mathcal{C}(\overline{\mathbb{B}})$. Now consider the $C^{*}$-algebra embedding (5) and define, for $s \in \partial \mathbb{B}$, a linear map

$$
\rho_{s}: \mathcal{A} \longrightarrow \mathbb{C} \oplus \mathbb{C}
$$

by putting

$$
\sigma_{s}\left(\begin{array}{ll}
A & B \\
C & D
\end{array}\right):=\left(\sigma_{s}^{\nu}(A), \sigma_{s}^{\nu+1}(D)\right) .
$$

This is in fact a $C^{*}$-algebra homomorphism since, for example, in an operator matrix product

$$
\left(\begin{array}{ll}
A_{1} & B_{1} \\
C_{1} & D_{1}
\end{array}\right)\left(\begin{array}{ll}
A_{2} & B_{2} \\
C_{2} & D_{2}
\end{array}\right)=\left(\begin{array}{ll}
A_{1} A_{2}+B_{1} C_{2} & A_{1} B_{2}+B_{1} D_{2} \\
C_{1} A_{2}+D_{1} C_{2} & C_{1} B_{2}+D_{1} D_{2}
\end{array}\right)
$$

the operators $B_{1} C_{2}$ and $C_{1} B_{2}$ are compact, and therefore

$$
\sigma_{s}^{\nu}\left(A_{1} A_{2}+B_{1} C_{2}\right)=\sigma_{s}^{\nu}\left(A_{1} A_{2}\right)=\sigma_{s}^{\nu}\left(A_{1}\right) \sigma_{s}^{\nu}\left(A_{2}\right)
$$


and, similarly,

$$
\sigma_{s}^{\nu+1}\left(C_{1} B_{2}+D_{1} D_{2}\right)=\sigma_{s}^{\nu+1}\left(D_{1} D_{2}\right)=\sigma_{s}^{\nu+1}\left(D_{1}\right) \sigma_{s}^{\nu+1}\left(D_{2}\right) .
$$

Now consider a generator $(4)$ of $\mathcal{T}_{\nu}\left(\mathbb{B}^{1 \mid 1}\right)$. Then we have

$$
\sigma_{s}\left(T_{F}^{(\nu)}\right)=\left(\sigma_{s}^{\nu}\left[T_{\nu}\left(f_{00}+\frac{1-w \bar{w}}{\nu-1} f_{11}\right)\right], \sigma_{s}^{\nu+1}\left[T_{\nu+1}\left(f_{00}\right)\right]\right)=\left(f_{00}(s), f_{00}(s)\right)
$$

since $1-w \bar{w}$ vanishes on $\partial \mathbb{B}$. It follows that $(7)$, when restricted to $\mathcal{T}_{\nu}\left(\mathbb{B}^{1 \mid 1}\right)$, has two identical entries. Identifying the two entries, we obtain the character (6).

Since the characters $\sigma_{s}^{\nu+i}(i=0,1)$ vanish on the ideal $\mathcal{K}_{\nu+i}=\mathcal{K}\left(H_{\nu+i}^{2}(\mathbb{B})\right)$ of all compact operators on $H_{\nu+i}^{2}(\mathbb{B})$, it is clear that $\sigma_{s}$ vanishes on

$$
\mathcal{K}\left(H_{\nu}^{2}\left(\mathbb{B}^{1 \mid 1}\right)\right)=\left(\begin{array}{cc}
\mathcal{K}_{\nu} & \mathcal{K}_{0}^{1} \\
\mathcal{K}_{1}^{0} & \mathcal{K}_{\nu+1}
\end{array}\right)
$$

Conversely, suppose an operator matrix

$$
\left(\begin{array}{ll}
A & B \\
C & D
\end{array}\right) \in \mathcal{T}_{\nu}\left(\mathbb{B}^{1 \mid 1}\right) \subset \mathcal{A}
$$

belongs to the joint null-space of $\sigma_{s}$ for all $s \in \partial \mathbb{B}$. According to (7) this means that

$$
\sigma_{s}^{\nu}(A)=0=\sigma_{s}^{\nu+1}(D)
$$

for all $s \in \partial \mathbb{B}$, since both entries in (7) are identical. Hence $A$ and $D$ are compact operators, and the assertion follows.

\section{Super-Bergman spaces on the unit ball}

For $p \geq 1$, let $\mathcal{O}\left(\mathbb{B}^{p}\right)$ denote the algebra of all holomorphic functions $\psi\left(z_{1}, \ldots, z_{p}\right)$ on the open unit ball

$$
\mathbb{B}^{p}:=\left\{z=\left(z_{1}, \ldots, z_{p}\right) \in \mathbb{C}^{p}:\|z\|^{2}=\sum_{i=1}^{p}\left\|z_{i}\right\|^{2}<1\right\}
$$

in $\mathbb{C}^{p}$. Let $\Lambda_{q}$ denote the complex Grassmann algebra with $q$ generators $\zeta_{1}, \ldots, \zeta_{q}$, satisfying the relations

$$
\zeta_{i} \zeta_{j}+\zeta_{j} \zeta_{i}=0
$$

for $1 \leq i, j \leq q$. Putting $Q:=\{1, \ldots, q\}$, we have

$$
\Lambda_{q}=\mathbb{C}\left\langle\zeta_{I}: I \subset Q\right\rangle
$$


where

$$
\zeta_{I}=\zeta_{i_{1}} \cdots \zeta_{i_{k}}
$$

if $I=\left\{i_{1}<\cdots<i_{k}\right\}$. For disjoint subsets $I, J$ we have

$$
\zeta_{I} \zeta_{J}=\varepsilon_{I, J} \zeta_{I \cup J}
$$

where $\varepsilon_{I, J}= \pm 1$. The tensor product algebra

$$
\mathcal{O}\left(\mathbb{B}^{p \mid q}\right):=\mathcal{O}\left(\mathbb{B}^{p}\right) \otimes \Lambda_{q}=\mathcal{O}\left(\mathbb{B}^{p}\right)\left\langle\zeta_{I}: I \subset Q\right\rangle
$$

consists of all "super-holomorphic" functions

$$
\Psi=\sum_{I \subset Q} \Psi_{I} \zeta_{I}
$$

where $\Psi_{I} \in \mathcal{O}\left(\mathbb{B}^{p}\right)$ for all $I \subset Q$. We sometimes write

$$
\Psi(z, \zeta)=\sum_{I \subset Q} \Psi_{I}(z) \zeta_{I}
$$

for all $z \in \mathbb{B}^{p}$. For $p=q=1$, we recover the situation of the unit disk.

Definition 3.1. For $\nu>p$, the weighted Bergman space

$$
H_{\nu}^{2}\left(\mathbb{B}^{p}\right):=\mathcal{O}\left(\mathbb{B}^{p}\right) \cap L^{2}\left(\mathbb{B}^{p}, d \mu_{\nu}\right)
$$

consists of all holomorphic functions on $\mathbb{B}^{p}$ which are square-integrable for the probability measure

$$
d \mu_{\nu}(z)=\frac{\Gamma(\nu)}{\pi^{p} \Gamma(\nu-p)}(1-(z \mid z))^{\nu-p-1} d z .
$$

Here $d z$ is the Lebesgue measure for the scalar product $(z \mid w)=\sum_{i} z_{i} \bar{w}_{i}$ on $\mathbb{C}^{p}$.

It is well-known [14] that $H_{\nu}^{2}\left(\mathbb{B}^{p}\right)$ has the reproducing kernel

$$
K_{\nu}(z, w)=(1-(z \mid w))^{-\nu}
$$

for all $z, w \in \mathbb{B}^{p}$. Let $\Lambda_{q}^{\mathbb{C}}$ denote the complex Grassmann algebra with $2 q$ generators $\bar{\zeta}_{1}, \ldots, \bar{\zeta}_{q}, \zeta_{1}, \ldots, \zeta_{q}$ satisfying

$$
\begin{aligned}
& \zeta_{i} \zeta_{j}+\zeta_{j} \zeta_{i}=0, \\
& \bar{\zeta}_{i} \bar{\zeta}_{j}+\bar{\zeta}_{j} \bar{\zeta}_{i}=0, \\
& \zeta_{i} \bar{\zeta}_{j}+\bar{\zeta}_{j} \zeta_{i}=0
\end{aligned}
$$

for all $1 \leq i, j \leq q$. Thus, putting $Q=\{1, \ldots, q\}$,

$$
\Lambda_{q}^{\mathbb{C}}=\mathbb{C}\left\langle\zeta_{I}^{*} \zeta_{J}: I, J \subset Q\right\rangle=\Lambda_{q}\left\langle\zeta_{I}^{*}: I \subset Q\right\rangle,
$$


where

$$
\zeta_{J}:=\zeta_{j_{1}} \cdots \zeta_{j_{\ell}}, \quad \zeta_{I}^{*}=\bar{\zeta}_{i_{k}} \cdots \bar{\zeta}_{i_{1}}
$$

for $I=\left\{i_{1}<\cdots<i_{k}\right\}, \quad J=\left\{j_{1}<\cdots<j_{\ell}\right\}$. The tensor product

$$
\mathcal{C}\left(\mathbb{B}^{p \mid q}\right):=\mathcal{C}\left(\mathbb{B}^{p}\right) \otimes \Lambda_{q}^{\mathbb{C}}=\mathcal{C}\left(\mathbb{B}^{p}\right)\left\langle\zeta_{I}^{*} \zeta_{J}: I, J \subset Q\right\rangle
$$

consists of all "continuous" super-functions

$$
F=\sum_{I, J} f_{I, J} \zeta_{I}^{*} \zeta_{J}
$$

where $f_{I, J} \in \mathcal{C}\left(\mathbb{B}^{p}\right)$ for all $I, J \subset Q$. The involution will be denoted by $F \mapsto F^{*}$. The Berezin integral on $\mathbb{B}^{p \mid q}$ is defined by

$$
\int_{\mathbb{B}^{p \mid q}} d z d \zeta F(z, \zeta)=\int_{\mathbb{B}^{p}} d z f_{Q, Q}(z)
$$

for $F \in \mathcal{C}\left(\mathbb{B}^{p \mid q}\right)$ as in $(9)$, where the normalization is given by

$$
\int_{\mathbb{C}^{0 \mid q}} d \zeta \zeta_{Q}^{*} \zeta_{Q}=\int_{\mathbb{C}^{0 \mid q}} d \zeta \prod_{j=1}^{q} \bar{\zeta}_{j} \zeta_{j}=1 .
$$

As in the case of the unit disk $(p=1)$ this unweighted integral is not suitable for quantization. Put

$$
\zeta \bar{\omega}=(\zeta \mid \omega)=\sum_{i} \zeta_{i} \bar{\omega}_{i} \in \Lambda_{q}^{\mathbb{C}}
$$

and

$$
z \bar{w}=(z \mid w)=\sum_{j=1}^{p} z_{j} \bar{w}_{j}
$$

Definition 3.2. For any parameter $\nu>p-q+1$, the (weighted) super-Bergman space

$$
H_{\nu}^{2}\left(\mathbb{B}^{p \mid q}\right) \subset \mathcal{O}\left(\mathbb{B}^{p \mid q}\right)
$$

consists of all super-holomorphic functions $\Psi(z, \zeta)$ which satisfy the square-integrability condition

$$
(\Psi \mid \Psi)_{\nu}:=\frac{\Gamma(\nu)}{\Gamma(\nu+q-p)} \frac{1}{\pi^{p}} \int_{\mathbb{B} p \mid q} d z d \zeta(1-z \bar{z}-\zeta \bar{\zeta})^{\nu+q-p-1} \Psi(z, \zeta)^{*} \Psi(z, \zeta)<+\infty .
$$


Lemma 3.3. Endow $\Lambda^{q}$ with a scalar product $(u \mid v)$, conjugate-linear in $u$, such that

$$
\zeta_{I}:=\prod_{i \in I} \zeta_{i}, \quad I \subset Q
$$

form an orthonormal basis. Then we have for $u, v \in \Lambda^{q}$

$$
\int_{\mathbb{C}^{0 \mid q}} d \zeta e^{-\zeta \bar{\zeta}} u^{*} v=(u \mid v)
$$

Proof. We have

$$
(\zeta \bar{\zeta})^{k}=\left(\zeta_{1} \bar{\zeta}_{1}+\cdots+\zeta_{q} \bar{\zeta}_{q}\right)^{k}=k ! \sum_{L} \prod_{\ell \in L} \zeta_{\ell} \bar{\zeta}_{\ell}
$$

where $L \subset Q$ has $k$ elements. Write $u=\sum_{I \subset Q} u_{I} \zeta_{I}, v=\sum_{J \subset Q} v_{J} \zeta_{J}$ with $u_{I}, v_{J} \in \mathbb{C}$. Then (10) implies

$$
e^{-\zeta \bar{\zeta}}=\sum_{k \geq 0} \frac{1}{k !}(-1)^{k}(\zeta \bar{\zeta})^{k}=\sum_{k=0}^{q}(-1)^{k} \sum_{\substack{L \subset Q \\|L|=k}} \prod_{\ell \in L} \zeta_{\ell} \bar{\zeta}_{\ell}=\sum_{L \subset Q} \prod_{\ell \in L} \bar{\zeta}_{\ell} \zeta_{\ell}=\sum_{L \subset Q} \zeta_{L}^{*} \zeta_{L}
$$

Using the fermionic integration rules it follows that

$$
\begin{aligned}
\int_{\mathbb{C}^{0 \mid q}} d \zeta e^{-\zeta \bar{\zeta}} u^{*} v & =\sum_{I, J} \bar{u}_{I} v_{J} \int_{\mathbb{C}^{0 \mid q}} d \zeta e^{-\zeta \bar{\zeta}} \zeta_{I}^{*} \zeta_{J} \\
& =\sum_{I, J} \bar{u}_{I} v_{J} \sum_{L} \int_{\mathbb{C}^{0 \mid q}} d \zeta \zeta_{L}^{*} \zeta_{L} \zeta_{I}^{*} \zeta_{J} \\
& =\sum_{L} \bar{u}_{Q \backslash L} v_{Q \backslash L}=(u \mid v) .
\end{aligned}
$$

Proposition 3.4.

$$
\frac{(1-z \bar{z}-\zeta \bar{\zeta})^{\nu+q-p-1}}{\Gamma(\nu+q-p)}=\sum_{J \subset Q} \frac{(1-z \bar{z})^{\nu+|J|-p-1}}{\Gamma(\nu+|J|-p)} \zeta_{Q \backslash J}^{*} \zeta_{Q \backslash J} .
$$


Proof. It follows from (10) that

$$
\begin{aligned}
(1-z \bar{z}-\zeta \bar{\zeta})^{\nu+q-p-1} & (1-z \bar{z})^{\nu+q-p-1}\left(1-\frac{\zeta \bar{\zeta}}{1-z \bar{z}}\right)^{\nu+q-p-1} \\
& =(1-z \bar{z})^{\nu+q-p-1} \sum_{k=0}^{q}\left(\begin{array}{c}
\nu+q-p-1 \\
q-k
\end{array}\right)(-1)^{q-k}(1-z \bar{z})^{k-q}(\zeta \bar{\zeta})^{q-k} \\
& =\sum_{k=0}^{q}\left(\begin{array}{c}
\nu+q-p-1 \\
q-k
\end{array}\right)(-1)^{q-k}(1-z \bar{z})^{\nu+k-p-1} \sum_{L \subset Q}(q-k) ! \prod_{\ell \in L} \zeta_{\ell} \bar{\zeta}_{\ell} \\
& =\sum_{k=0}^{q}(1-z \bar{z})^{\nu+k-p-1} \frac{\Gamma(\nu+q-p)}{\Gamma(\nu+k-p)} \sum_{|L|=q-k} \prod_{\ell \in L} \bar{\zeta}_{\ell} \zeta_{\ell} .
\end{aligned}
$$

Putting $J=Q \backslash L$, the assertion follows.

Proposition 3.5. For $\Psi=\sum_{M \subset Q} \psi_{M} \zeta_{M} \in \mathcal{O}\left(\mathbb{B}^{p \mid q}\right)$ we have

$$
\begin{aligned}
& \frac{\Gamma(\nu)}{\Gamma(\nu+q-p)} \frac{1}{\pi^{p}} \int_{\mathbb{B}^{p \mid q}} d z d \zeta(1-z \bar{z}-\zeta \bar{\zeta})^{\nu+q-p-1} \Psi^{*}(z, \zeta) \Psi(z, \zeta) \\
& =\sum_{m=0}^{q} \frac{\Gamma(\nu)}{\Gamma(\nu+m)} \sum_{\substack{M \subset Q \\
|M|=m}} \frac{\Gamma(\nu+m)}{\Gamma(\nu+m-p)} \frac{1}{\pi^{p}} \int_{\mathbb{B}^{p}} d z(1-z \bar{z})^{\nu+m-p-1} \cdot \overline{\psi_{M}(z)} \psi_{M}(z),
\end{aligned}
$$

i.e., there is an orthogonal decomposition

$$
H_{\nu}^{2}\left(\mathbb{B}^{p \mid q}\right)=\sum_{m=0}^{q} H_{\nu+m}^{2}\left(\mathbb{B}^{p}\right) \otimes \Lambda^{m}\left(\mathbb{C}^{q}\right)
$$

into a sum of weighted Bergman spaces for $0 \leq m \leq q$, with multiplicity $\left(\begin{array}{c}q \\ m\end{array}\right)$.

Proof. For $\Psi=\sum_{M \subset Q} \psi_{M} \zeta_{M}$ we have, according to Proposition 3.4,

$$
\begin{aligned}
S:= & \frac{\Gamma(\nu)}{\Gamma(\nu+q-p)} \frac{1}{\pi^{p}} \int_{\mathbb{B}^{p \mid q}} d z d \zeta(1-z \bar{z}-\zeta \bar{\zeta})^{\nu+q-p-1} \Psi^{*}(z, \zeta) \Psi(z, \zeta) \\
= & \sum_{K \subset Q} \sum_{I \subset Q} \sum_{J \subset Q} \frac{\Gamma(\nu)}{\Gamma(\nu+|K|-p)} \frac{1}{\pi^{p}} \int_{\mathbb{B}^{p}} d z(1-z \bar{z})^{\nu+|K|-p-1} \\
& \times \overline{\psi_{I}(z)} \psi_{J}(z) \int_{\mathbb{C} 0 \mid q} d \zeta \zeta_{Q \backslash K}^{*} \zeta_{Q \backslash K} \zeta_{I}^{*} \zeta_{J} .
\end{aligned}
$$


The fermionic integration rules imply that only the choice $I=K=J$ contributes, and hence

$$
S=\sum_{M} \frac{\Gamma(\nu)}{\Gamma(\nu+|M|-p)} \frac{1}{\pi^{p}} \int_{\mathbb{B}^{p}} d z(1-z \bar{z})^{\nu+|M|-p-1} \overline{\psi_{M}(z)} \psi_{M}(z) .
$$

\section{Proposition 3.6.}

$$
\frac{\Gamma(\nu)}{(1-z \bar{w}-\zeta \bar{\omega})^{\nu}}=\sum_{I \subset Q} \frac{\Gamma(\nu+|I|)}{(1-z \bar{w})^{\nu+|I|}} \prod_{i \in I} \zeta_{i} \bar{\omega}_{i}
$$

Proof. We have

$$
\begin{aligned}
(1-z \bar{w}-\zeta \bar{\omega})^{-\nu} & =(1-z \bar{w})^{-\nu}\left(1-\frac{\zeta \bar{\omega}}{1-z \bar{w}}\right)^{-\nu} \\
& =(1-z \bar{w})^{-\nu} \sum_{i=0}^{q}\left(\begin{array}{c}
-\nu \\
i
\end{array}\right)(-1)^{i}\left(\frac{\zeta \bar{\omega}}{1-z \bar{w}}\right)^{i} \\
& =\sum_{i=0}^{q}\left(\begin{array}{c}
-\nu \\
i
\end{array}\right)(-1)^{i}(1-z \bar{w})^{-\nu-i}(\zeta \bar{\omega})^{i} \\
& =\sum_{i=0}^{q}\left(\begin{array}{c}
-\nu \\
i
\end{array}\right)(-1)^{i}(1-z \bar{w})^{-\nu-i} \sum_{I \subset Q} i ! \prod_{k \in I} \zeta_{k} \bar{\omega}_{k} \\
& =\sum_{i=0}^{q}(1-z \bar{w})^{-\nu-i} \frac{\Gamma(\nu+i)}{\Gamma(\nu)} \sum_{|I|=i} \prod_{k \in I} \zeta_{k} \bar{\omega}_{k} .
\end{aligned}
$$

Proposition 3.7. For $\nu>p$ and $\Psi=\sum_{M} \psi_{M} \zeta_{M} \in H_{\nu}^{2}\left(\mathbb{B}^{p \mid q}\right)$ we have the reproducing kernel property

$$
\begin{aligned}
& \Psi(z, \zeta) \\
& \quad=\frac{\Gamma(\nu)}{\Gamma(\nu+q-p)} \frac{1}{\pi^{p}} \int_{\mathbb{B}^{p \mid q}} d w d \omega(1-w \bar{w}-\omega \bar{\omega})^{\nu+q-p-1}(1-z \bar{w}-\zeta \bar{\omega})^{-\nu} \Psi(w, \omega),
\end{aligned}
$$

i.e., $H_{\nu}^{2}\left(\mathbb{B}^{p \mid q}\right)$ has the reproducing kernel

$$
K_{\nu}(z, \zeta, w, \omega)=(1-z \bar{w}-\zeta \bar{\omega})^{-\nu}
$$


Proof. Applying Proposition 3.4 and Proposition 3.6, we obtain for $\nu>p$

$$
\begin{aligned}
S:= & \frac{\Gamma(\nu)}{\Gamma(\nu+q-p)} \frac{1}{\pi^{p}} \\
& \times \int_{\mathbb{B}^{p}} d w \int_{\mathbb{C}^{0 \mid q}} d \omega(1-w \bar{w}-\omega \bar{\omega})^{\nu+q-p-1}(1-z \bar{w}-\zeta \bar{\omega})^{-\nu} \sum_{M \subset Q} \psi_{M}(w) \omega_{M} \\
= & \sum_{M \subset Q} \sum_{I \subset Q} \sum_{J \subset Q} \frac{\Gamma(\nu+|I|)}{\Gamma(\nu+|J|-p)} \frac{1}{\pi^{p}} \int_{\mathbb{B}^{p}} d w(1-w \bar{w})^{\nu+|J|-p-1}(1-z \bar{w})^{-\nu-|I|} \psi_{M}(w) \\
& \times \int_{\mathbb{C}^{0 \mid q}} d \omega \omega_{Q \backslash J}^{*} \omega_{Q \backslash J} \prod_{i \in I} \zeta_{i} \bar{\omega}_{i} \omega_{M} .
\end{aligned}
$$

The fermionic integration rules imply that only the choices $J=M=I$ contribute. It follows that

$$
S=\sum_{I} \zeta_{I} \frac{\Gamma(\nu+|I|)}{\Gamma(\nu+|I|-p)} \frac{1}{\pi^{p}} \int_{\mathbb{B}^{p}} d w(1-w \bar{w})^{\nu+|I|-p-1}(1-z \bar{w})^{-\nu-|I|} \psi_{I}(w)
$$

since

$$
\prod_{i \in I} \zeta_{i} \bar{\omega}_{i} \omega_{I}=\omega_{I}^{*} \omega_{I} \zeta_{I}
$$

Now apply the reproducing property for each weighted Bergman space $H_{\nu+|I|}^{2}\left(\mathbb{B}^{p}\right)$ with $\nu+|I| \geq \nu>p$.

\section{Super-Toeplitz operators on the unit ball}

For $F \in \mathcal{C}\left(\overline{\mathbb{B}}^{p \mid q}\right)$, the super-Toeplitz operator $T_{F}^{(\nu)}$ on $H_{\nu}^{2}\left(\mathbb{B}^{p \mid q}\right)$ is defined as

$$
T_{F}^{(\nu)} \Psi=P^{(\nu)}(F \Psi),
$$

where $P^{(\nu)}$ denotes the orthogonal projection onto $H_{\nu}^{2}\left(\mathbb{B}^{p \mid q}\right)$.

Proposition 4.1. With respect to the decomposition $\Psi=\sum_{M} \psi_{M} \zeta_{M}$, the superToeplitz operator $T_{F}^{(\nu)}$ on $H_{\nu}^{2}\left(\mathbb{B}^{p \mid q}\right)$ is given by the $2^{q} \times 2^{q}$-matrix

$$
\begin{aligned}
\left(T_{F}^{(\nu)}\right)_{I}^{J}=\sum_{I \cup J \subset K \subset Q} \varepsilon_{K \backslash I, I} \varepsilon_{K \backslash J, J} & \\
& \times \frac{\Gamma(\nu+|I|-p)}{\Gamma(\nu+|K|-p)} T_{\nu+|I|}^{\nu+|J|}\left(F_{K \backslash I, K \backslash J}(w) \cdot(1-w \bar{w})^{|K|-|I|}\right) .
\end{aligned}
$$


Here, for $0 \leq i, j \leq q, T_{\nu+i}^{\nu+j}$ denotes the Bergman-type Toeplitz operator

$$
T_{\nu+i}^{\nu+j}(f)=P_{\nu+i} f P_{\nu+j}^{*}: H_{\nu+j}^{2}\left(\mathbb{B}^{p}\right) \longrightarrow H_{\nu+i}^{2}\left(\mathbb{B}^{p}\right)
$$

from $H_{\nu+j}^{2}\left(\mathbb{B}^{p}\right)$ to $H_{\nu+i}^{2}\left(\mathbb{B}^{p}\right)$.

Proof. Using Proposition 3.4 and Proposition 3.6 we obtain

$$
\begin{aligned}
S:= & \left(T_{F} \Psi\right)(z, \zeta) \\
= & \frac{\Gamma(\nu)}{\Gamma(\nu+q-p)} \frac{1}{\pi^{p}} \int_{\mathbb{B}^{p}} d w \int_{\mathbb{C}^{0 \mid q}} d \omega(1-w \bar{w}-\omega \bar{\omega})^{\nu+q-p-1}(1-z \bar{w}-\zeta \bar{\omega})^{-\nu} \\
& \quad \times \sum_{M, N} f_{M, N}(w) \omega_{M}^{*} \omega_{N} \sum_{J \subset Q} \psi_{J}(w) \omega_{J} \\
= & \sum_{I \subset Q} \sum_{K \subset Q} \sum_{J \subset Q} \frac{\Gamma(\nu+|I|)}{\Gamma(\nu+|K|-p)} \frac{1}{\pi^{p}} \\
& \quad \times \int_{\mathbb{B} p} d w(1-w \bar{w})^{\nu+|K|-p-1}(1-z \bar{w})^{-\nu-|I|} \\
& \quad \times \sum_{M, N} f_{M, N}(w) \psi_{J}(w) \int_{\mathbb{C}^{0 \mid q}} d \omega \omega_{Q \backslash K}^{*} \omega_{Q \backslash K} \prod_{i \in I} \zeta_{i} \bar{\omega}_{i} \omega_{M}^{*} \omega_{N} \omega_{J} .
\end{aligned}
$$

The fermionic integration rules imply that non-zero terms occur only when $K=M \cup I$ $=N \cup J$ as disjoint unions. Therefore

$$
\begin{aligned}
S:= & \sum_{I \subset Q} \sum_{J \subset Q} \sum_{I \cup J \subset K} \frac{\Gamma(\nu+|I|)}{\Gamma(\nu+|K|-p)} \frac{1}{\pi^{p}} \int_{\mathbb{B}^{p}} d w(1-w \bar{w})^{\nu+|K|-p-1}(1-z \bar{w})^{-\nu-|I|} \\
& \times f_{K \backslash I, K \backslash J}(w) \psi_{J}(w) \int_{\mathbb{C}^{0 \mid q}} d \omega \omega_{Q \backslash K}^{*} \omega_{Q \backslash K} \prod_{i \in I} \zeta_{i} \bar{\omega}_{i} \omega_{K \backslash I}^{*} \omega_{K \backslash J} \omega_{J} \\
= & \sum_{I \cup J \subset K} \frac{\Gamma(\nu+|I|)}{\Gamma(\nu+|K|-p)} \frac{1}{\pi^{p}} \int_{\mathbb{B}^{p}} d w(1-w \bar{w})^{\nu+|K|-p-1}(1-z \bar{w})^{-\nu-|I|} \\
& \times f_{K \backslash I, K \backslash J}(w) \psi_{J}(w) \int_{\mathbb{C}^{0 \mid q}} d \omega \omega_{Q \backslash K}^{*} \omega_{Q \backslash K} \prod_{i \in I} \zeta_{i} \bar{\omega}_{i} \omega_{K \backslash I}^{*} \omega_{K} \varepsilon_{K \backslash J, J .}
\end{aligned}
$$

In view of (9) we have

$$
\begin{aligned}
\prod_{i \in I} \zeta_{i} \bar{\omega}_{i} \omega_{K \backslash I}^{*} \omega_{K} & =\varepsilon_{K \backslash I, I} \prod_{i \in I} \zeta_{i} \bar{\omega}_{i} \omega_{K \backslash I}^{*} \omega_{K \backslash I} \omega_{I}=\varepsilon_{K \backslash I, I} \omega_{K \backslash I}^{*} \omega_{K \backslash I} \prod_{i \in I} \zeta_{i} \bar{\omega}_{i} \omega_{I} \\
& =\varepsilon_{K \backslash I, I} \omega_{K \backslash I}^{*} \omega_{K \backslash I} \omega_{I}^{*} \omega_{I} \zeta_{I}=\varepsilon_{K \backslash I, I} \omega_{K}^{*} \omega_{K} \zeta_{I} .
\end{aligned}
$$


It follows that

$$
\begin{aligned}
& S= \sum_{I \cup J \subset K} \varepsilon_{K \backslash I, I} \varepsilon_{K \backslash J, J} \frac{\Gamma(\nu+|I|)}{\Gamma(\nu+|K|-p)} \frac{1}{\pi^{p}} \int_{\mathbb{B}^{p}} d w(1-w \bar{w})^{\nu+|K|-p-1}(1-z \bar{w})^{-\nu-|I|} \\
& \times f_{K \backslash I, K \backslash J}(w) \psi_{J}(w) \int_{\mathbb{C}^{0 \mid q}} d \omega \omega_{Q \backslash K}^{*} \omega_{Q \backslash K} \omega_{K}^{*} \omega_{K} \zeta_{I} \\
&= \sum_{I \cup J \subset K} \varepsilon_{K \backslash I, I} \varepsilon_{K \backslash J, J} \frac{\Gamma(\nu+|I|)}{\Gamma(\nu+|K|-p)} \frac{1}{\pi^{p}} \int_{\mathbb{B}^{p}} d w(1-w \bar{w})^{\nu+|K|-p-1}(1-z \bar{w})^{-\nu-|I|} \\
& \times f_{K \backslash I, K \backslash J}(w) \psi_{J}(w) \int_{\mathbb{C}^{0 \mid q}} d \omega \omega_{Q}^{*} \omega_{Q} \zeta_{I} \\
&=\sum_{I \cup J \subset K} \varepsilon_{K \backslash I, I} \varepsilon_{K \backslash J, J} \frac{\Gamma(\nu+|I|)}{\Gamma(\nu+|K|-p)} \frac{1}{\pi^{p}} \int_{\mathbb{B}^{p}} d w(1-w \bar{w})^{\nu+|K|-p-1}(1-z \bar{w})^{-\nu-|I|} \\
& \quad \times f_{K \backslash I, K \backslash J}(w) \psi_{J}(w) \zeta_{I} \\
&=\sum_{I \cup J \subset K} \varepsilon_{K \backslash I, I} \varepsilon_{K \backslash J, J} \frac{\Gamma(\nu+|I|-p)}{\Gamma(\nu+|K|-p)} \frac{\Gamma(\nu+|I|)}{\Gamma(\nu+|I|-p)} \frac{1}{\pi^{p}} \\
& \quad \times \int_{\mathbb{B}{ }^{p}} d w(1-w \bar{w})^{\nu+|I|-p-1}(1-z \bar{w})^{-\nu-|I|} \\
& \quad \times f_{K \backslash I, K \backslash J}(w)(1-w \bar{w})^{|K|-|I|} \\
& \quad \psi_{J}(w) \zeta_{I} \cdot
\end{aligned}
$$

Since, for $\psi \in H_{\nu+j}^{2}\left(\mathbb{B}^{p}\right)$,

$$
\left(T_{\nu+i}^{\nu+j}(f) \psi\right)(z)=\frac{\Gamma(\nu+i)}{\Gamma(\nu+i-p)} \frac{1}{\pi^{p}} \int_{\mathbb{B}^{p}} d w(1-w \bar{w})^{\nu+i-p-1}(1-z \bar{w})^{-\nu-i} f(w) \psi(w)
$$

the assertion follows.

Theorem 4.2. The $C^{*}$-algebra $\mathcal{T}_{\nu}\left(\mathbb{B}^{p \mid q}\right)$, generated by all Toeplitz operators with symbols $F \in \mathcal{C}\left(\overline{\mathbb{B}}^{p \mid q}\right)$ which are continuous up to the boundary, can be embedded in the $(q+1) \times(q+1)$-matrix operator algebra

$$
\mathcal{T}_{\nu}\left(\mathbb{B}^{p \mid q}\right) \subset\left(\mathcal{A}_{i}^{j}\left(\mathbb{B}^{p}\right)\right)_{0 \leq i, j \leq q}=: \mathcal{A},
$$

where for $i \neq j$ we have

$$
\mathcal{A}_{i}^{j}\left(\mathbb{B}^{p}\right)=\left\{\text { compact operators } H_{\nu+j}^{2}\left(\mathbb{B}^{p}\right) \otimes \Lambda^{j}\left(\mathbb{C}^{q}\right) \longrightarrow H_{\nu+i}^{2}\left(\mathbb{B}^{p}\right) \otimes \Lambda^{i}\left(\mathbb{C}^{q}\right)\right\},
$$

whereas for $i=j$ we have

$$
\mathcal{A}_{i}^{i}\left(\mathbb{B}^{p}\right)=\mathcal{T}_{\nu+i}\left(\mathbb{B}^{p}\right) \otimes \operatorname{End}\left(\Lambda^{i}\left(\mathbb{C}^{q}\right)\right) .
$$

Here $\mathcal{T}_{\nu+i}\left(\mathbb{B}^{p}\right)$ denotes the Toeplitz $C^{*}$-algebra on $H_{\nu+i}^{2}\left(\mathbb{B}^{p}\right)$. 
Proof. For $0 \leq i, j \leq q$ the matrix block of $\mathcal{T}_{F}^{(\nu)}$, from $H_{\nu+j}^{2}\left(\mathbb{B}^{p}\right)$ to $H_{\nu+i}^{2}\left(\mathbb{B}^{p}\right)$, has the operator entries

$$
\left(\mathcal{T}_{F}^{(\nu)}\right)_{I}^{J}, \quad|I|=i, \quad|J|=j,
$$

given explicitly by (11). For $i=j$, these operator entries belong to the Toeplitz $C^{*}$-algebra $\mathcal{T}_{\nu+i}\left(\mathbb{B}^{p}\right)$. In order to prove Theorem 4.2 it suffices to show that for $i \neq j$ these entries are compact operators.

In the case $i>j$, the Toeplitz type operator $T_{\nu+i}^{\nu+j}(f)$ from $H_{\nu+j}^{2}\left(\mathbb{B}^{p}\right)$ to $H_{\nu+i}^{2}\left(\mathbb{B}^{p}\right)$ is given by the composition $P \circ M_{f}$ where $P$ is the restriction of the Bergman projection $P_{\nu+i}: L_{\nu+i}^{2}\left(\mathbb{B}^{p}\right) \rightarrow H_{\nu+i}^{2}\left(\mathbb{B}^{p}\right)$ to the space $L_{\nu+j}^{2}\left(\mathbb{B}^{p}\right)$. Using [14, Theorem 2.10], we see that the restriction $P$ is a continuous operator from $L_{\nu+j}^{2}\left(\mathbb{B}^{p}\right)$ to $H_{\nu+j}^{2}\left(\mathbb{B}^{p}\right)$. Since the inclusion of $H_{\nu+j}^{2}\left(\mathbb{B}^{p}\right)$ in the space $H_{\nu+i}^{2}\left(\mathbb{B}^{p}\right)$ is a compact operator it follows that the Toeplitz type operator $T_{\nu+i}^{\nu+j}$ is compact.

Now suppose $|I|<|J|$. In this case we have $|K|>|I|$ whenever $K \supset I \cup J$. It follows that the corresponding operator of multiplication by the function $(1-z \bar{z})^{|K|-|I|}$ from $H_{\nu+j}^{2}\left(\mathbb{B}^{p}\right)$ to $L_{\nu+j}^{2}\left(\mathbb{B}^{p}\right)$ is a compact operator since $|K|-|I| \geq 1$. Then the operator $M_{f}$ is compact, implying that

$$
\left(\mathcal{T}_{F}^{(\nu)}\right)_{I}^{J}, \quad|I|=i, \quad|J|=j,
$$

is compact. These arguments establish the embedding (12) on the level of generators. Since the right-hand side of (12) is a $C^{*}$-algebra, the assertion follows.

The following Theorem, generalizing Theorem 2.3, is the first main result of this paper.

Theorem 4.3. For every boundary point $s \in \partial \mathbb{B}^{p}=\mathbb{S}^{2 p-1}$, there exists a $C^{*}$-algebra homomorphism (character)

$$
\sigma_{s}: \mathcal{T}_{\nu}\left(\mathbb{B}^{p \mid q}\right) \longrightarrow \mathbb{C}
$$

that is uniquely determined by the property

$$
\sigma_{s}\left(\mathcal{T}_{F}^{(\nu)}\right)=F_{\emptyset, \emptyset}(s)
$$

for $F=\sum_{I, J} F_{I, J} \zeta_{I}^{*} \zeta_{J}$ and $F_{I, J} \in \mathcal{C}\left(\overline{\mathbb{B}}^{p}\right)$ continuous up to the boundary. Moreover, the joint null-space

$$
\bigcap_{s \in \partial \mathbb{B}^{p}} \operatorname{Ker} \sigma_{s}=\mathcal{K}\left(H_{\nu}^{2}\left(\mathbb{B}^{p \mid q}\right)\right)
$$

of all these characters coincides with the ideal of all compact operators on the superBergman space $H_{\nu}^{2}\left(\mathbb{B}^{p \mid q}\right)$.

Proof. Since the Toeplitz $C^{*}$-algebra $\mathcal{T}_{\nu}\left(\mathbb{B}^{p}\right)$ acts irreducibly on $H_{\nu}^{2}\left(\mathbb{B}^{p}\right)$ and has a nontrivial intersection with the compact operators $\mathcal{K}\left(H_{\nu}^{2}\left(\mathbb{B}^{p}\right)\right)$, it follows that $\mathcal{K}\left(H_{\nu}^{2}\left(\mathbb{B}^{p}\right)\right)$ is contained in $\mathcal{T}_{\nu}\left(\mathbb{B}^{p}\right)$. Applying [10, Theorem 4.12.32] to the special case of the unit 
ball $\mathbb{B}^{p}$ and the Bergman-Toeplitz operators for parameters $\nu+i, 0 \leq i \leq q$, we obtain $C^{*}$-algebra homomorphisms

$$
\sigma_{s}^{\nu+i}: \mathcal{T}_{\nu+i}\left(\mathbb{B}^{p}\right) \longrightarrow \mathbb{C}
$$

whose kernel is the ideal of compact operators and satisfy

$$
\sigma_{s}^{\nu+i}\left(T_{f}^{\nu+i}\right)=f(s)
$$

whenever $f \in \mathcal{C}\left(\overline{\mathbb{B}}^{p}\right)$. Now consider the $C^{*}$-algebra embedding (12) and define, for $s \in \partial \mathbb{B}^{p}$, a linear map

$$
\sigma_{s}: \mathcal{A} \longrightarrow \mathbb{C}^{2^{q}}
$$

by putting

$$
\sigma_{s}\left(T_{I}^{J}\right)=\left(\sigma_{s}^{\nu+|I|}\left(T_{I}^{I}\right)\right)_{I \subset Q} .
$$

Since, by assumption, the off-diagonal entries $T_{I}^{J}$ are compact operators and $\sigma_{s}^{\nu+i}$ vanishes on $\mathcal{K}\left(H_{\nu+i}^{2}\left(\mathbb{B}^{p}\right)\right)$, a computation analogous to (8) shows that (14) is in fact a $C^{*}$-algebra homomorphism.

Now consider a generator $T_{F}^{(\nu)}$ of $\mathcal{T}_{\nu}\left(\mathbb{B}^{p \mid q}\right)$. Using its matrix representation (11) it follows that

$$
\begin{aligned}
\sigma_{s}^{\nu+|I|}\left(\left(T_{F}^{(\nu)}\right)_{I}^{I}\right) & =\sum_{I \subset K \subset Q} \frac{\Gamma(\nu+|I|-p)}{\Gamma(\nu+|K|-p)} \sigma_{s}^{\nu+|I|}\left(T^{\nu+|I|}\left(F_{K \backslash I, K \backslash I}(w)(1-w \bar{w})^{|K|-|I|}\right)\right) \\
& =\sigma_{s}^{\nu+|I|}\left(T^{\nu+|I|}\left(F_{\emptyset, \emptyset}\right)\right)=F_{\emptyset, \emptyset}(s),
\end{aligned}
$$

since for $K \neq I$ we have $|K|-|I| \geq 1$ and the symbol function $F_{K \backslash I, K \backslash I}(w)$ $(1-w \bar{w})^{|K|-|I|}$ vanishes at $s \in \partial \mathbb{B}^{p}$. It follows that $(14)$, when restricted to $\mathcal{T}_{\nu}\left(\mathbb{B}^{p \mid q}\right)$, has only identical entries for all $I \subset Q$. Identifying these entries, we obtain the character (13).

Since the characters $\sigma_{s}^{\nu+i}(0 \leq i \leq q)$ vanish on the ideal $\mathcal{K}_{\nu+i}=\mathcal{K}\left(H_{\nu+i}^{2}\left(\mathbb{B}^{p}\right)\right)$ of all compact operators on $H_{\nu+i}^{2}\left(\mathbb{B}^{p}\right)$, it is clear that $\sigma_{s}$ vanishes on the ideal

$$
\mathcal{K}\left(H_{\nu}^{2}\left(\mathbb{B}^{p \mid q}\right)\right)=\left\{\left(T_{I}^{J}\right)_{I, J \subset Q} \in \mathcal{A}: T_{I}^{I} \in \mathcal{K}_{\nu+|I|} \quad \text { for all } I \subset Q\right\}
$$

of $\mathcal{A}$. Conversely, suppose that an operator matrix $\left(T_{I}^{J}\right)_{I, J \subset Q}$ in $\mathcal{T}_{\nu}\left(\mathbb{B}^{p \mid q}\right) \subset \mathcal{A}$ belongs to the joint null-space of $\sigma_{s}$ for all $s \in \partial \mathbb{B}^{p}$. According to (14), this means

$$
\sigma_{s}^{\nu+|I|}\left(T_{I}^{I}\right)=0
$$

for all $I \subset Q$ since all entries in (14) are identical. Thus $T_{I}^{I} \in \mathcal{K}_{\nu+|I|}$ are compact operators, and the assertion follows. 


\section{Super-Bergman spaces on matrix balls}

For $1 \leq r \leq p$, let $\mathcal{O}\left(\mathbb{B}^{r \times p}\right)$ denote the algebra of all holomorphic functions

$$
\psi\left(z_{1}^{1}, \ldots, z_{1}^{p}, \ldots, z_{r}^{1}, \ldots, z_{r}^{p}\right)=\psi\left(z_{i}^{j}\right)
$$

on the matrix ball

$$
\mathbb{B}^{r \times p}=\left\{z=\left(z_{i}^{j}\right)_{\substack{1 \leq i \leq r \\ 1 \leq j \leq p}} \in \mathbb{C}^{r \times p}: 1-z z^{*}>0\right\}
$$

in $\mathbb{C}^{r \times p}$. For fixed $q \geq 1$, let $\Lambda^{r \times q}$ denote the complex Grassmann algebra with $r \cdot q$ generators $\zeta_{1}^{1}, \ldots, \zeta_{1}^{q}, \ldots, \zeta_{r}^{1}, \ldots, \zeta_{r}^{q}$ satisfying the relations

$$
\zeta_{i}^{j} \zeta_{k}^{\ell}+\zeta_{k}^{\ell} \zeta_{i}^{j}=0
$$

for all $1 \leq i, k \leq r$ and $1 \leq j, \ell \leq q$. Putting $R:=\{1, \ldots, r\}$, we have

$$
\Lambda^{r \times q}=\mathbb{C}\left\langle\zeta_{M}: M \subset R \times Q\right\rangle,
$$

where

$$
\zeta_{M}=\prod_{(i, j) \in M} \zeta_{i}^{j}
$$

and the index-pairs $(i, j)$ are ordered lexicographically:

$$
(i, j)<(k, \ell) \Longleftrightarrow i<k \quad \text { or } \quad i=k, j<\ell .
$$

The tensor product algebra

$$
\mathcal{O}\left(\mathbb{B}^{r \times p \mid r \times q}\right)=\mathcal{O}\left(\mathbb{B}^{r \times p}\right) \otimes \Lambda^{r \times q}:=\mathcal{O}\left(\mathbb{B}^{r \times p}\right)\left\langle\zeta_{M}: M \subset R \times Q\right\rangle
$$

consists of all "super-holomorphic" functions

$$
\Psi=\sum_{M \subset R \times Q} \psi_{M} \zeta_{M}
$$

where $\psi_{M} \in \mathcal{O}\left(\mathbb{B}^{r \times p}\right)$ for all $M \subset R \times Q$. We sometimes write

$$
\Psi(z, \zeta)=\sum_{M \subset R \times Q} \psi_{M}(z) \zeta_{M}
$$

for all $z \in \mathbb{B}^{r \times p}$. The case $r=1$ corresponds to the unit ball.

Definition 5.1. For $\nu>r+p-1$, the weighted Bergman space

$$
H_{\nu}^{2}\left(\mathbb{B}^{r \times p}\right):=\mathcal{O}\left(\mathbb{B}^{r \times p}\right) \cap L^{2}\left(\mathbb{B}^{r \times p}, d \mu_{\nu}\right)
$$


consists of all holomorphic functions on $\mathbb{B}^{r \times p}$ which are square-integrable for the probability measure

$$
d \mu_{\nu}(z)=\frac{1}{\pi^{r p}} \prod_{j=1}^{r} \frac{\Gamma(\nu+1-j)}{\Gamma(\nu+1-j-p)} \operatorname{det}\left(1-z z^{*}\right)^{\nu-r-p} d z .
$$

Here $d z$ is the Lebesgue measure for the scalar product $(z \mid w):=\operatorname{tr} z w^{*}$ on $\mathbb{C}^{r \times p}$.

It is well-known that $H_{\nu}^{2}\left(\mathbb{B}^{r \times p}\right)$ has the reproducing kernel

$$
K_{\nu}(z, w)=\operatorname{det}\left(1-z w^{*}\right)^{-\nu}
$$

for all $z, w \in \mathbb{B}^{r \times p}$. Let $\Lambda_{r \times q}^{\mathbb{C}}$ denote the complex Grassmann algebra with $2 r q$ generators $\bar{\zeta}_{i}^{j}, \zeta_{k}^{\ell}(1 \leq i, k \leq r, 1 \leq j, \ell \leq q)$ satisfying the relations (15) and, in addition,

$$
\bar{\zeta}_{i}^{j} \bar{\zeta}_{k}^{\ell}+\bar{\zeta}_{k}^{\ell} \bar{\zeta}_{i}^{j}=0=\bar{\zeta}_{i}^{j} \zeta_{k}^{\ell}+\zeta_{k}^{\ell} \bar{\zeta}_{i}^{j} .
$$

Defining

$$
\zeta_{M}^{*}:=\prod_{(i, j) \in M} \bar{\zeta}_{i}^{j}
$$

for the reverse lexicographic order (cf. (16)), we thus have

$$
\Lambda_{r \times q}^{\mathbb{C}}=\mathbb{C}\left\langle\zeta_{M}^{*} \zeta_{N}: M, N \subset R \times Q\right\rangle=\Lambda_{r \times q}\left\langle\zeta_{M}^{*}: M \subset R \times Q\right\rangle .
$$

The tensor product

$$
\mathcal{C}\left(\mathbb{B}^{r \times p \mid r \times q}\right):=\mathbb{C}\left(\mathbb{B}^{r \times p}\right) \otimes \Lambda_{r \times q}^{\mathbb{C}}=\mathbb{C}\left(\mathbb{B}^{r \times p}\right)\left\langle\zeta_{M}^{*} \zeta_{N}: M, N \subset R \times Q\right\rangle
$$

consists of all "continuous" super-functions

$$
F=\sum_{M, N} f_{M, N} \zeta_{M}^{*} \zeta_{N}
$$

where $f_{M, N} \in \mathcal{C}\left(\mathbb{B}^{r \times p}\right)$ for all $M, N \subset R \times Q$. The Berezin integral on $\mathbb{B}^{r \times p \mid r \times q}$ is defined by

$$
\int_{\mathbb{B}^{r \times p \mid r \times q}} d z d \zeta F(z, \zeta)=\int_{\mathbb{B}^{r \times p}} d z F_{R \times Q}, R \times Q(z)
$$

for $F \in \mathcal{C}\left(\mathbb{B}^{r \times p \mid r \times q}\right)$ as in (17), where the normalization is given by

$$
\int_{\mathbb{C}^{|| r \times q}} d \zeta \zeta_{R \times Q}^{*} \zeta_{R \times Q}=\int_{\mathbb{C}^{0 \mid r \times q}} d \zeta \prod_{i=1}^{r} \prod_{j=1}^{q} \bar{\zeta}_{i}^{j} \zeta_{i}^{j}=1 .
$$


Definition 5.2. For any parameter $\nu>p+r-q+1$, the (weighted) super-Bergman space

$$
H_{\nu}^{2}\left(\mathbb{B}^{r \times p \mid r \times q}\right) \subset \mathcal{O}\left(\mathbb{B}^{r \times p \mid r \times q}\right)
$$

consists of all super-holomorphic functions $\Psi(z, \zeta)$ which satisfy the square-integrability condition

$$
\begin{aligned}
(\Psi \mid \Psi)_{\nu}:= & \frac{1}{\pi^{r p}} \prod_{j=1}^{r} \frac{\Gamma(\nu+1-j)}{\Gamma(\nu+1-j+q-p)} \\
& \times \int_{\mathbb{B}^{r \times p \mid r \times q}} d z d \zeta \operatorname{det}\left(1-z z^{*}-\zeta \zeta^{*}\right)^{\nu+q-p-r} \cdot \Psi(z, \zeta)^{*} \Psi(z, \zeta)<+\infty .
\end{aligned}
$$

The main result of this section, generalizing Proposition 1.3 (for the unit disk) and Proposition 3.5 (for the unit ball), is an explicit orthogonal decomposition of the (scalar-valued) super-Bergman space $H_{\nu}^{2}\left(\mathbb{B}^{r \times p \mid r \times q}\right)$ as a direct sum of "ordinary" weighted Bergman spaces over the matrix ball $\mathbb{B}^{r \times p}$. It turns out that for domains of higher rank $r>1$ it is not enough to consider scalar-valued Bergman spaces, as introduced in Definition 5.1, but vector-valued holomorphic functions arise in a canonical way in the decomposition of $H_{\nu}^{2}\left(\mathbb{B}^{r \times p \mid r \times q}\right)$. These vector-valued functions are parameterized by partitions of length smaller than or equal to $r$ and, as an interesting feature, the associated multiplicity space corresponds exactly to the conjugate partition.

A partition of length smaller than or equal to $r$ is a non-increasing sequence of integers

$$
\boldsymbol{m}=m_{1} \geq m_{2} \geq \cdots \geq m_{r} \geq 0 .
$$

It is well-known [5] that partitions classify irreducible (finite-dimensional) representations of the matrix group $\mathrm{GL}_{r}(\mathbb{C})$. A canonical model for the corresponding representation space is given by the Schur functor denoted by $\mathbb{S}_{\boldsymbol{m}}\left(\mathbb{C}^{r}\right)$. Let

$$
\pi_{m}: \mathrm{GL}_{r}(\mathbb{C}) \rightarrow \mathrm{GL}\left(\mathbb{S}_{\boldsymbol{m}}\left(\mathbb{C}^{r}\right)\right)
$$

be the corresponding representation, and choose an inner product $(u \mid v)_{\boldsymbol{m}}$ on $\mathbb{S}_{\boldsymbol{m}}\left(\mathbb{C}^{r}\right)$, conjugate-linear in $u$, which is invariant under the unitary subgroup $U(r)$. Graphically, a partition $\boldsymbol{m}$ is represented by its Young diagram

$$
\left\{(i, j) \mid 1 \leq i \leq r, \quad 1 \leq j \leq m_{i}\right\} .
$$

We will mainly consider partitions satisfying the additional requirement $m_{1} \leq q$, i.e.,

$$
q \geq m_{1} \geq m_{2} \geq \cdots \geq m_{r} \geq 0 .
$$

In this case the Young diagram is contained in the rectangle

$$
R \times Q=\{(i, j) \mid 1 \leq i \leq r, \quad 1 \leq j \leq q\}
$$


It is easy to show that there exist exactly $\left(\begin{array}{c}r+q \\ r\end{array}\right)$ partitions $\boldsymbol{m}$ of this type. Every partition $\boldsymbol{m}$ has a conjugate partition $\boldsymbol{m}^{\sharp}$ obtained by transposing its Young diagram. If (19) holds, the conjugate partition $\boldsymbol{m}^{\sharp}$ has length $\leq q$, so we have the corresponding Schur functor $\mathbb{S}_{m}^{\sharp}\left(\mathbb{C}^{q}\right)$ and the associated representation $\pi_{m}^{\sharp}$ of $\mathrm{GL}_{q}(\mathbb{C})$.

Definition 5.3. For any partition

$$
\boldsymbol{m}=m_{1} \geq \cdots \geq m_{r} \geq 0
$$

of length smaller than or equal to $r$, and $\nu>r+p-1$, the vector-valued Bergman space

$$
H_{\nu}^{2}\left(\mathbb{B}^{r \times p}, \mathbb{S}_{\boldsymbol{m}}\left(\mathbb{C}^{r}\right)\right)
$$

consists of all holomorphic maps

$$
\psi: \mathbb{B}^{r \times p} \longrightarrow \mathbb{S}_{\boldsymbol{m}}\left(\mathbb{C}^{r}\right)
$$

into the Schur functor representation space, which satisfy the square-integrability condition

$$
\begin{aligned}
(\psi \mid \psi)_{\nu, \boldsymbol{m}}= & \frac{1}{\pi^{r p}} \prod_{j=1}^{r} \frac{\Gamma(\nu+1-j)}{\Gamma(\nu+1-j-p)} \\
& \times \int_{\mathbb{B}^{r \times p}} d z \operatorname{det}\left(1_{r}-z z^{*}\right)^{\nu-r-p} \cdot\left(\psi(z) \mid \pi_{\boldsymbol{m}}\left(1_{r}-z z^{*}\right) \psi(z)\right)_{\boldsymbol{m}}<+\infty .
\end{aligned}
$$

Note that $1_{r}-z z^{*} \in \mathrm{GL}_{r}(\mathbb{C})$ for any $z \in \mathbb{B}^{r \times p}$, making $\pi_{\boldsymbol{m}}\left(1_{r}-z z^{*}\right) \in \mathrm{GL}\left(\mathbb{S}_{\boldsymbol{m}}\left(\mathbb{C}^{r}\right)\right)$ well-defined. It is well-known that a similar construction yields the so-called holomorphic discrete series of the group $U(r, p)$, and other semi-simple Lie groups of Hermitian type.

Our main result concerning Bergman spaces of super-holomorphic functions on the matrix ball is the following

Theorem 5.4. For $\nu>r+p-q-1$, the super-Bergman space $H_{\nu}^{2}\left(\mathbb{B}^{r \times p \mid r \times q}\right)$ introduced in Definition 5.2 has an orthogonal decomposition

$$
H_{\nu}^{2}\left(\mathbb{B}^{r \times p \mid r \times q}\right)=\sum_{\boldsymbol{m} \subset R \times Q} H_{\nu}^{2}\left(\mathbb{B}^{r \times p}, \mathbb{S}_{\boldsymbol{m}}\left(\mathbb{C}^{r}\right)\right) \otimes \mathbb{S}_{\boldsymbol{m}}^{\sharp}\left(\mathbb{C}^{q}\right)
$$

where $\boldsymbol{m}$ runs over all partitions

$$
q \geq m_{1} \geq \cdots \geq m_{r} \geq 0
$$

with Schur functor $\mathbb{S}_{\boldsymbol{m}}\left(\mathbb{C}^{r}\right)$, and $\mathbb{S}_{\boldsymbol{m}}^{\sharp}\left(\mathbb{C}^{q}\right)$ is the Schur functor representation space associated with the conjugate partition $\boldsymbol{m}^{\sharp}$ of length smaller than or equal to $q$. 
Remark 5.5. For rank $r=1$ and $0 \leq m \leq q$, the conjugate partition

$$
m^{\sharp}=1^{m} 0^{q-m}
$$

has the Schur functor $\mathbb{S}_{m}^{\sharp}\left(\mathbb{C}^{q}\right)=\Lambda^{m}\left(\mathbb{C}^{q}\right)$. Since $\mathbb{S}_{m}^{\sharp}\left(\mathbb{C}^{1}\right) \equiv \mathbb{C}$ in this case, we recover the decomposition (10) for the unit ball.

Proof of Theorem 5.4. Identify

$$
\Lambda^{r \times q}=\Lambda\left(\mathbb{C}^{r \times q}\right)=\Lambda\left(\mathbb{C}^{r} \otimes \mathbb{C}^{q}\right)
$$

with the exterior algebra over $\mathbb{C}^{r \times q}=\mathbb{C}^{r} \otimes \mathbb{C}^{q}$, and consider the canonical action of $\mathrm{GL}_{r}(\mathbb{C}) \times \mathrm{GL}_{q}(\mathbb{C})$ on $\Lambda^{r \times q}$ induced by left and right matrix multiplication

$$
\pi(g, h) \zeta:=g \zeta h^{-1}
$$

for $g \in \mathrm{GL}_{r}(\mathbb{C}), h \in \mathrm{GL}_{q}(\mathbb{C})$, and $\zeta \in \mathbb{C}^{r \times q}$. A basic result in combinatorial harmonic analysis $[5$, Exercise 6.11 , p. 80$]$ asserts that there is a multiplicity-free decomposition

$$
\Lambda\left(\mathbb{C}^{r \times q}\right)=\sum_{\boldsymbol{m} \subset R \times Q} \mathbb{S}_{\boldsymbol{m}}\left(\mathbb{C}^{r}\right) \otimes \mathbb{S}_{\boldsymbol{m}}^{\sharp}\left(\mathbb{C}^{q}\right)
$$

into irreducible $\mathrm{GL}_{r}(\mathbb{C}) \times \mathrm{GL}_{q}(\mathbb{C})$-submodules, where $\boldsymbol{m}=m_{1} \geq \cdots \geq m_{r} \geq 0$ runs over all partitions of length smaller than or equal to $r$ satisfying $m_{1} \leq q$, and the associated Schur functor representations are defined as above. For $g \in \mathrm{GL}_{r}(\mathbb{C})$ and $h \in \mathrm{GL}_{q}(\mathbb{C})$, it follows that

$$
\pi(g, h)(a \otimes b)=\pi_{\boldsymbol{m}}(g) a \otimes \pi_{\boldsymbol{m}}^{\sharp}(h) b
$$

whenever $a \in \mathbb{S}_{\boldsymbol{m}}\left(\mathbb{C}^{r}\right)$ and $b \in \mathbb{S}_{\boldsymbol{m}}^{\sharp}\left(\mathbb{C}^{q}\right)$. For $\zeta=\left(\zeta_{i}^{j}\right) \in \mathbb{C}^{r \times q}$, with adjoint matrix $\zeta^{*} \in \mathbb{C}^{q \times r}$, we have

$$
\operatorname{tr} \zeta \zeta^{*}=\sum_{i=1}^{r} \sum_{j=1}^{q} \zeta_{i}^{j} \bar{\zeta}_{i}^{j} .
$$

Applying Lemma 3.3 to the index set $R \times Q$ (endowed with the lexicographic total ordering) we obtain for the Berezin integral

$$
\int_{\mathbb{C}^{0 \mid r \times q}} d \zeta e^{-\operatorname{tr} \zeta \zeta^{*}} u^{*} v=(u \mid v)
$$

for any $u, v \in \Lambda^{r \times q}$, where $(u \mid v)$ is the inner product defined as in Lemma 3.3. This inner product is invariant under the action

$$
\zeta \longmapsto g \zeta h^{*}
$$


of $(g, h) \in U(r) \times U(q)$ on $\Lambda^{r \times q}$. Since the decomposition (21) is multiplicity free, it follows that

$$
(u \mid v)=\sum_{\boldsymbol{m} \subset R \times Q}\left(u_{\boldsymbol{m}} \mid v_{\boldsymbol{m}}\right)_{\boldsymbol{m}, \boldsymbol{m}^{\sharp}}
$$

whenever $u=\sum_{m} u_{m}$ and $v=\sum_{m} v_{m}$, with components $u_{m}, v_{m} \in \mathbb{S}_{m}\left(\mathbb{C}^{r}\right) \otimes$ $\mathbb{S}_{\boldsymbol{m}}^{\sharp}\left(\mathbb{C}^{q}\right)$. For any $\boldsymbol{m}$ the $U(r) \times U(q)$-invariant inner product $(\mid)_{\boldsymbol{m}, \boldsymbol{m}^{\sharp}}$ on $\mathbb{S}_{\boldsymbol{m}}\left(\mathbb{C}^{r}\right) \otimes$ $\mathbb{S}_{m^{\sharp}}\left(\mathbb{C}^{q}\right)$ is a tensor product

$$
(a \otimes b \mid c \otimes d)_{\boldsymbol{m}, \boldsymbol{m}^{\sharp}}=(a \mid c)_{\boldsymbol{m}}(b \mid d)_{\boldsymbol{m}}^{\sharp}
$$

of a $U(r)$-invariant inner product $(\mid)_{\boldsymbol{m}}$ on $\mathbb{S}_{\boldsymbol{m}}\left(\mathbb{C}^{r}\right)$ and a $U(q)$-invariant inner product $(\mid)_{m}^{\sharp}$ on $\mathbb{S}_{m}^{\sharp}\left(\mathbb{C}^{q}\right)$. For a matrix variable $\zeta \in \mathbb{C}^{r \times q}$, the Fischer-Fock reproducing kernel functions $K_{m}(\zeta, \zeta)[7]$ are defined by the expansion

$$
e^{\operatorname{tr} \zeta \zeta^{*}}=\sum_{m} K_{m}(\zeta, \zeta)
$$

where $\boldsymbol{m}=m_{1} \geq \cdots \geq m_{r} \geq 0$ runs over all partitions of length $\leq r$. Moreover, one can express

$$
K_{\boldsymbol{m}}(\zeta, \zeta)=\sum_{i} u_{i}(\zeta) u_{i}(\zeta)^{*}
$$

in terms of an orthonormal basis $u_{i}$ in the associated $U(r) \times U(q)$-submodule $\mathcal{P}_{\boldsymbol{m}}\left(\mathbb{C}^{r \times q}\right)$ of polynomials on $\mathbb{C}^{r \times q}$. (For details, see [7] or [10].) Now assume that $\zeta=\left(\zeta_{i}^{j}\right)$ is a "Grassmann" matrix, satisfying the relations (15). Define

$$
K_{m}(\zeta, \zeta) \in \Lambda_{r \times q}^{\mathbb{C}}
$$

by (26), using the involution ${ }^{*}$ of $\Lambda_{r \times q}^{\mathbb{C}}$. Then the Grassmann relations (15) imply that $K_{\boldsymbol{m}}(\zeta, \zeta)=0$ unless $m_{1} \leq q$, i.e., $\boldsymbol{m} \subset R \times Q$. Thus in this case (25) becomes

$$
e^{-\operatorname{tr} \zeta \zeta^{*}}=\sum_{\boldsymbol{m} \subset R \times Q}(-1)^{|\boldsymbol{m}|} K_{\boldsymbol{m}}(\zeta, \zeta),
$$

where $|\boldsymbol{m}|:=m_{1}+\cdots+m_{r}$. In view of (22) and (23), this implies

$$
\int_{\mathbb{C}^{0 \mid r \times q}} d \zeta K_{\boldsymbol{m}}(\zeta, \zeta) u^{*} v=(-1)^{|\boldsymbol{m}|}\left(u_{\boldsymbol{m}} \mid v_{\boldsymbol{m}}\right)_{\boldsymbol{m}, \boldsymbol{m}^{*}}
$$

for all $u, v \in \Lambda^{r \times q}$ and $\boldsymbol{m} \subset R \times Q$. Thus the projection operators associated with the decomposition (21) can be expressed via the Berezin integral. The Faraut-Korányi binomial expansion [6], for matrices $\zeta \in \mathbb{C}^{r \times q}$, yields the formula

$$
\operatorname{det}\left(1_{r}-\zeta \zeta^{*}\right)^{\alpha}=\sum_{m}(-\alpha)_{m} K_{m}(\zeta, \zeta)
$$


where

$$
(-\alpha)_{\boldsymbol{m}}=\prod_{i=1}^{r}(-\alpha+1-i)_{\boldsymbol{m}_{i}}=\prod_{i=1}^{r} \prod_{j=1}^{m_{i}}(j-i-\alpha)
$$

is the multivariable Pochhammer symbol. Note that

$$
(-1)^{|\boldsymbol{m}|}(-\alpha)_{\boldsymbol{m}}=\prod_{i=1}^{r} \prod_{j=1}^{m_{i}}(\alpha+i-j) .
$$

Imposing the Grassmann relations (15), it follows that only partitions $\boldsymbol{m} \subset R \times Q$ occur, and we obtain

$$
\operatorname{det}\left(1_{r}-\zeta \zeta^{*}\right)^{\alpha}=\sum_{m \subset R \times Q}(-\alpha)_{m} K_{m}(\zeta, \zeta) .
$$

Therefore (27) implies for all $u, v \in \Lambda^{r \times q}$

$$
\begin{aligned}
\int_{\mathbb{C}^{0 \mid r \times q}} d \zeta \operatorname{det}\left(1_{r}-\zeta \zeta^{*}\right)^{\alpha} u^{*} v & =\sum_{\boldsymbol{m} \subset R \times Q}(-\alpha)_{\boldsymbol{m}} \int_{\mathbb{C}^{0 \mid r \times q}} d \zeta K_{\boldsymbol{m}}(\zeta, \zeta) u^{*} v \\
& =\sum_{\boldsymbol{m} \subset R \times Q}(-\alpha)_{\boldsymbol{m}}(-1)^{|\boldsymbol{m}|}\left(u_{\boldsymbol{m}} \mid v_{\boldsymbol{m}}\right)_{\boldsymbol{m}, \boldsymbol{m}^{\sharp}} \\
& =\sum_{\boldsymbol{m} \subset R \times Q} \prod_{i=1}^{r} \prod_{j=1}^{m_{i}}(\alpha+i-j)\left(u_{\boldsymbol{m}} \mid v_{\boldsymbol{m}}\right)_{\boldsymbol{m}, \boldsymbol{m}^{\sharp} .}
\end{aligned}
$$

To finish the proof of Theorem 5.4, let

$$
\Psi(z, \zeta) \in \mathcal{O}\left(\mathbb{B}^{r \times p \mid r \times q}\right)=\mathcal{O}\left(\mathbb{B}^{r \times p}\right) \otimes \Lambda^{r \times q}
$$

be a super-holomorphic function, and consider its canonical decomposition

$$
\Psi(z, \zeta)=\sum_{m \subset R \times Q} \Psi_{m}(z)
$$

with $\Psi_{\boldsymbol{m}}(z) \in \mathbb{S}_{\boldsymbol{m}}\left(\mathbb{C}^{r}\right) \otimes \mathbb{S}_{\boldsymbol{m}}^{\sharp}\left(\mathbb{C}^{q}\right)$, according to $(21)$. For any $z \in \mathbb{B}^{r \times p}$ consider the fermionic change of variables

$$
\zeta=\left(1_{r}-z z^{*}\right)^{1 / 2} \vartheta
$$

Since

$$
\begin{aligned}
1_{r}-z z^{*}-\zeta \zeta^{*} & =1_{r}-z z^{*}-\left(1_{r}-z z^{*}\right)^{1 / 2} \vartheta \vartheta^{*}\left(1_{r}-z z^{*}\right)^{1 / 2} \\
& =\left(1_{r}-z z^{*}\right)^{1 / 2}\left(1_{r}-\vartheta \vartheta^{*}\right)\left(1_{r}-z z^{*}\right)^{1 / 2}
\end{aligned}
$$


it follows that

$$
\operatorname{det}\left(1_{r}-z z^{*}-\zeta \zeta^{*}\right)=\operatorname{det}\left(1_{r}-z z^{*}\right) \operatorname{det}\left(1_{r}-\vartheta \vartheta^{*}\right)
$$

Therefore the fermionic integral transformation formula [3] yields

$$
\begin{aligned}
& \int_{\mathbb{B}^{r \times p \mid r \times q}} d z d \zeta \operatorname{det}\left(1_{r}-z z^{*}-\zeta \zeta^{*}\right)^{\nu+q-p-r} \Psi(z, \zeta)^{*} \Psi(z, \zeta) \\
&=\int_{\mathbb{B}^{r \times p}} d z \operatorname{det}\left(1_{r}-z z^{*}\right)^{\nu+2 q-p-r} \int_{\mathbb{C}^{0 \mid r \times q}} d \vartheta \operatorname{det}\left(1_{r}-\vartheta \vartheta^{*}\right)^{\nu+q-p-r} \\
& \times \Psi\left(z,\left(1_{r}-z z^{*}\right)^{1 / 2} \vartheta\right)^{*} \Psi\left(z,\left(1_{r}-z z^{*}\right)^{1 / 2} \vartheta\right) .
\end{aligned}
$$

Since $\left(1_{r}-z z^{*}\right)^{1 / 2}$ acts only on the left, its effect on the decomposition (29) is given by

$$
\Psi\left(z,\left(1_{r}-z z^{*}\right)^{1 / 2} \vartheta\right)=\sum_{\boldsymbol{m} \subset R \times Q} \pi_{\boldsymbol{m}}\left(\left(1_{r}-z z^{*}\right)^{1 / 2}, 1_{q}\right) \Psi_{\boldsymbol{m}}(z) .
$$

Applying (28) to $\alpha:=\nu+q-p-r$ and $u=v:=\Psi\left(z,\left(1_{r}-z z^{*}\right)^{1 / 2} \vartheta\right) \in \Lambda^{r \times q}(\vartheta)$ we obtain, using the self-adjointness of $1_{r}-z z^{*} \in \mathrm{GL}_{r}(\mathbb{C})$,

$$
\begin{aligned}
& \int_{\mathbb{C}^{0 \mid r \times q}} d \vartheta \operatorname{det}\left(1-\vartheta \vartheta^{*}\right)^{\nu+q-p-r} \Psi\left(z,\left(1_{r}-z z^{*}\right)^{1 / 2} \vartheta\right)^{*} \Psi\left(z,\left(1_{r}-z z^{*}\right)^{1 / 2} \vartheta\right) \\
& =\sum_{\boldsymbol{m} \subset R \times Q} \prod_{i=1}^{r} \prod_{j=1}^{m_{i}}(\nu+q-p-r+i-j) \\
& \quad \times\left(\pi_{\boldsymbol{m}}\left(\left(1_{r}-z z^{*}\right)^{1 / 2}, 1_{q}\right) \Psi_{\boldsymbol{m}}(z) \mid \pi_{\boldsymbol{m}}\left(\left(1_{r}-z z^{*}\right)^{1 / 2}, 1_{q}\right) \Psi_{\boldsymbol{m}}(z)\right)_{\boldsymbol{m}, \boldsymbol{m}^{\sharp}} \\
& =\sum_{\boldsymbol{m} \subset R \times Q} \prod_{i=1}^{r} \prod_{j=1}^{m_{i}}(\nu+q-p-r+i-j) \\
& \quad \times\left(\Psi_{\boldsymbol{m}}(z) \mid \pi_{\boldsymbol{m}}\left(1_{r}-z z^{*}, 1_{q}\right) \Psi_{\boldsymbol{m}}(z)\right)_{\boldsymbol{m}, \boldsymbol{m}^{\sharp}}
\end{aligned}
$$


Combining (18), (30), and (31) we obtain

$$
\begin{aligned}
& (\Psi \mid \Psi)_{\nu} \\
& =\frac{1}{\pi^{r p}} \prod_{j=1}^{r} \frac{\Gamma(\nu+1-j)}{\Gamma(\nu+1-j+q-p)} \int_{\mathbb{B}^{r \times p}} d z \operatorname{det}\left(1_{r}-z z^{*}\right)^{\nu+2 q-p-r} \\
& \quad \times \int_{\mathbb{C}^{0 \mid r \times q}} d \vartheta \operatorname{det}\left(1_{r}-\vartheta \vartheta^{*}\right)^{\nu+q-p-r} \Psi\left(z,\left(1_{r}-z z^{*}\right)^{1 / 2} \vartheta\right)^{*} \Psi\left(z,\left(1_{r}-z z^{*}\right)^{1 / 2} \vartheta\right) \\
& =\frac{1}{\pi^{r p}} \prod_{j=1}^{r} \frac{\Gamma(\nu+1-j)}{\Gamma(\nu+1-j+q-p)} \sum_{\boldsymbol{m} \subset R \times Q} \prod_{i=1}^{r} \prod_{j=1}^{m_{i}}(\nu+q-p-r+i-j) \\
& \quad \times \int_{\mathbb{B}^{r \times p}} d z \operatorname{det}\left(1_{r}-z z^{*}\right)^{\nu+2 q-p-r}\left(\Psi_{\boldsymbol{m}}(z) \mid \pi_{\boldsymbol{m}}\left(1_{r}-z z^{*}, 1_{q}\right) \Psi_{\boldsymbol{m}}(z)\right)_{\boldsymbol{m}, \boldsymbol{m}^{\sharp} .}
\end{aligned}
$$

For each $\boldsymbol{m} \subset R \times Q$, choose an orthonormal basis $B_{\boldsymbol{m}}$ of $\mathbb{S}_{\boldsymbol{m}}^{\sharp}\left(\mathbb{C}^{q}\right)$ and write

$$
\Psi_{\boldsymbol{m}}(z)=\sum_{b \in B_{m}} \psi_{\boldsymbol{m}}^{b}(z) \otimes b
$$

where $\psi_{m}^{b}: \mathbb{B}^{r \times p} \rightarrow \mathbb{S}_{\boldsymbol{m}}\left(\mathbb{C}^{r}\right)$ is holomorphic. Since $\pi_{\boldsymbol{m}}\left(1_{r}-z z^{*}, 1_{q}\right)=$ $\pi_{\boldsymbol{m}}\left(1_{r}-z z^{*}\right) \otimes$ id acts only on the left factor of $\mathbb{S}_{\boldsymbol{m}}\left(\mathbb{C}^{r}\right) \otimes \mathbb{S}_{\boldsymbol{m}}^{\sharp}\left(\mathbb{C}^{q}\right)$ we have

$$
\pi_{\boldsymbol{m}}\left(1_{r}-z z^{*}, 1_{q}\right) \Psi_{\boldsymbol{m}}(z)=\sum_{b \in B_{m}} \pi_{\boldsymbol{m}}\left(1_{r}-z z^{*}\right) \psi_{\boldsymbol{m}}^{b}(z) \otimes b
$$

and therefore, using (24) and orthonormality of $B_{\boldsymbol{m}}$

$$
\left(\Psi_{\boldsymbol{m}}(z) \mid \pi_{\boldsymbol{m}}\left(1_{r}-z z^{*}, 1_{q}\right) \Psi_{\boldsymbol{m}}(z)\right)_{\boldsymbol{m}, \boldsymbol{m}^{\sharp}}=\sum_{b \in B_{\boldsymbol{m}}}\left(\psi_{\boldsymbol{m}}^{b}(z) \mid \pi_{\boldsymbol{m}}\left(1_{r}-z z^{*}\right) \psi_{\boldsymbol{m}}^{b}(z)\right)_{\boldsymbol{m}} .
$$

In terms of the inner product (20) for the vector-valued Bergman space we obtain

$$
\begin{aligned}
\int_{\mathbb{B}^{r \times p}} d z \operatorname{det} & \left(1_{r}-z z^{*}\right)^{\nu+2 q-p-r}\left(\Psi_{\boldsymbol{m}}(z) \mid \pi_{\boldsymbol{m}}\left(1_{r}-z z^{*}, 1_{q}\right) \Psi_{\boldsymbol{m}}(z)\right)_{\boldsymbol{m}, \boldsymbol{m}}^{\sharp} \\
= & \sum_{b \in B_{\boldsymbol{m}}} \int_{\mathbb{B}^{r \times p}} d z \operatorname{det}\left(1_{r}-z z^{*}\right)^{\nu+2 q-p-r}\left(\Psi_{\boldsymbol{m}}^{b}(z) \mid \pi_{\boldsymbol{m}}\left(1_{r}-z z^{*}\right) \psi_{\boldsymbol{m}}^{b}(z)\right)_{\boldsymbol{m}} \\
= & \sum_{b \in B_{\boldsymbol{m}}}\left(\psi_{\boldsymbol{m}}^{b} \mid \psi_{\boldsymbol{m}}^{b}\right)_{\nu+2 q, \boldsymbol{m}}=\left(\Psi_{\boldsymbol{m}} \mid \Psi_{\boldsymbol{m}}\right)_{H_{\nu+2 q}^{2}\left(\mathbb{B}^{r \times p}, \mathbb{S}_{\boldsymbol{m}}\left(\mathbb{C}^{r}\right)\right) \otimes \mathbb{S}_{\boldsymbol{m}}^{\sharp}\left(\mathbb{C}^{q}\right)^{.}}
\end{aligned}
$$

In view of (32), the assertion follows. 
Acknowledgement. The authors would like to thank the referee for valuable remarks.

\section{References}

[1] F. A. Berezin, Introduction to superanalysis, with a foreword by A. A. Kirillov and an appendix by V. I. Ogievetsky, Mathematical Physics and Applied Mathematics, vol. 9, D. Reidel Publishing Co., Dordrecht, 1987.

[2] D. Borthwick, S. Klimek, A. Lesniewski, and M. Rinaldi, Super-Toeplitz operators and nonperturbative deformation quantization of supermanifolds, Comm. Math. Phys. 153 (1993), no. 1, 49-76.

[3] _ Matrix Cartan superdomains, super Toeplitz operators, and quantization, J. Funct. Anal. 127 (1995), no. 2, 456-510.

[4] D. Borthwick, A. Lesniewski, and H. Upmeier, Nonperturbative deformation quantization of Cartan domains, J. Funct. Anal. 113 (1993), no. 1, 153-176.

[5] W. Fulton and J. Harris, Representation theory, Graduate Texts in Mathematics, vol. 129, Springer-Verlag, New York, 1991. A first course; Readings in Mathematics.

[6] J. Faraut and A. Korányi, Function spaces and reproducing kernels on bounded symmetric domains, J. Funct. Anal. 88 (1990), no. 1, 64-89.

[7] Analysis on symmetric cones, Oxford Mathematical Monographs, Clarendon Press, Oxford, 1994

[8] M. B. Green, J. H. Schwarz, and E. Witten, Superstring theory, Cambridge Monographs on Mathematical Physics, Cambridge University Press, Cambridge, 1988.

[9] H. Hedenmalm, B. Korenblum, and K. Zhu, Theory of Bergman spaces, Graduate Texts in Mathematics, vol. 199, Springer-Verlag, New York, 2000

[10] H. Upmeier, Toeplitz operators and index theory in several complex variables, Operator Theory: Advances and Applications, vol. 81, Birkhäuser Verlag, Basel, 1996.

[11] Jordan algebras and harmonic analysis on symmetric spaces, Amer. J. Math. 108 (1986), no. 1, 1-25.

[12] _ Toeplitz operators on bounded symmetric domains, Trans. Amer. Math. Soc. 280 (1983), no. 1, 221-237

[13] _ Toeplitz $C^{*}$-algebras on bounded symmetric domains, Ann. of Math. (2) 119 (1984), no. $3,549-576$.

[14] K. Zhu, Spaces of holomorphic functions in the unit ball, Graduate Texts in Mathematics, vol. 226, Springer-Verlag, New York, 2005. 